\title{
Heterogeneity in endothelial cells and widespread venous arterialization during early vascular development in mammals
}

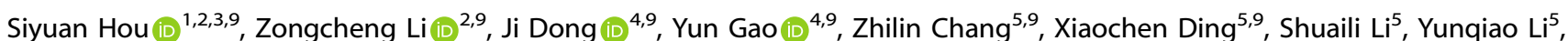 \\ Yang Zeng ${ }^{2}$, Qian Xin ${ }^{5}$, Baihan Wang ${ }^{5}$, Yanli $\mathrm{Ni}^{2}$, Xiaowei Ning ${ }^{5}$, Yuqiong $\mathrm{Hu}^{4}$, Xiaoying Fan (1) ${ }^{4}$, Yu Hou (10) ${ }^{4}$, Xianlong $\mathrm{Li}^{2}$, Lu Wen ${ }^{4,6}$, \\ Bin Zhou $\mathbb{D}^{7}$, Bing Liu ${ }^{1,2,5 凶}$, Fuchou Tang $\mathbb{( D )}^{4,6,8}$ and Yu Lan (10) ${ }^{1 凶}$
}

(c) The Author(s) 2022

\begin{abstract}
Arteriogenesis rather than unspecialized capillary expansion is critical for restoring effective circulation to compromised tissues in patients. Deciphering the origin and specification of arterial endothelial cells during embryonic development will shed light on the understanding of adult arteriogenesis. However, during early embryonic angiogenesis, the process of endothelial diversification and molecular events underlying arteriovenous fate settling remain largely unresolved in mammals. Here, we constructed the single-cell transcriptomic landscape of vascular endothelial cells (VECS) during the time window for the occurrence of key vasculogenic and angiogenic events in both mouse and human embryos. We uncovered two distinct arterial VEC types, the major artery VECs and arterial plexus VECs, and unexpectedly divergent arteriovenous characteristics among VECs that are located in morphologically undistinguishable vascular plexus intra-embryonically. Using computational prediction and further lineage tracing of venous-featured VECs with a newly developed $\mathrm{Nr}_{2} \mathrm{f2}^{\mathrm{CrexER}}$ mouse model and a dual recombinase-mediated intersectional genetic approach, we revealed early and widespread arterialization from the capillaries with considerable venous characteristics. Altogether, our findings provide unprecedented and comprehensive details of endothelial heterogeneity and lineage relationships at early angiogenesis stages, and establish a new model regarding the arteriogenesis behaviors of early intra-embryonic vasculatures.
\end{abstract}

Cell Research (2022) 32:333-348; https://doi.org/10.1038/s41422-022-00615-z

\section{INTRODUCTION}

During mammalian embryogenesis, the cardiovascular system is the first functional organ system to form. Vascular endothelial cells (VECs) undergo de novo differentiation from mesodermal precursors and assemble into a primordial vascular network through vasculogenesis. During the establishment of vasculature, VECs are needed to grow new vessels from pre-existing ones via sprouting angiogenesis, while the immature vascular plexus undergoes further remodeling to form recognizable arteries and veins and build a hierarchically organized vascular network. ${ }^{1-4}$ Arteriogenesis refers to the formation of new arteries and arterioles either de novo by means of capillary arterialization or by growth from pre-existing arterial collaterals, which is crucial in embryonic vascular development as well as in adult tissues. ${ }^{5}$ The growth of arteries but not capillaries has been demonstrated to be the key to restoring effective circulation to compromised tissues. ${ }^{6}$ Unfortunately, due to the insufficient understanding of arterial specification and arterial conduit formation, the field of therapeutic angiogenesis has not made significant progress in the past decade. Studies of cellular and molecular events underlying the specification of arterial VECs during embryonic development will help to understand adult arteriogenesis.

In developmental setting, arteries can form in multiple ways, ${ }^{6}$ with cell fate conversion playing a role under certain circumstances. In mice, endocardial cells contribute to a substantial proportion of postnatal coronary arteries through de novo lineage conversion during trabecular compaction. ${ }^{7}$ New arteries can also be generated from venous VECs. During hindbrain vascularization in zebrafish, artery forms by medial sprouting and migration of endothelial cells from a bilateral pair of primitive veins, that is, the primordial hindbrain channels. ${ }^{8,9}$ During mouse development, the coronary arteries of the heart are initially formed by venous VECs from the sinus venosus. ${ }^{10,11}$ In the postnatal mouse retina, veinderived endothelial tip cells continuously migrate from veins to arterial areas and contribute to emerging arteries. ${ }^{3,12}$ Notably, although the conversion of venous VECs into arteries is observed during the later stages of organogenesis and in tissue regeneration, it is not thought to occur during the expansion of the early

\footnotetext{
${ }^{1}$ Key Laboratory for Regenerative Medicine of Ministry of Education, Institute of Hematology, School of Medicine, Jinan University, Guangzhou, Guangdong, China. ${ }^{2}$ State Key Laboratory of Experimental Hematology, Haihe Laboratory of Cell Ecosystem, Institute of Hematology, Fifth Medical Center of Chinese PLA General Hospital, Beijing, China. ${ }^{3}$ Integrated Chinese and Western Medicine Postdoctoral Research Station, Jinan University, Guangzhou, Guangdong, China. ${ }^{4}$ Beijing Advanced Innovation Center for Genomics and Biomedical Pioneering Innovation Center, School of Life Sciences, Peking University, Beijing, China. ${ }^{5}$ State Key Laboratory of Proteomics, Academy of Military Medical Sciences, Academy of Military Sciences, Beijing, China. ${ }^{6}$ Ministry of Education Key Laboratory of Cell Proliferation and Differentiation, Beijing, China. ${ }^{7}$ The State Key Laboratory of Cell Biology, CAS Center for Excellence in Molecular Cell Science, Shanghai Institute of Biochemistry and Cell Biology, Chinese Academy of Sciences, University of Chinese Academy of Sciences, Shanghai, China. ${ }^{8}$ Peking-Tsinghua Center for Life Sciences, Peking University, Beijing, China. ${ }^{9}$ These authors contributed equally: Siyuan Hou, Zongcheng Li, Ji Dong, Yun Gao, Zhilin Chang, Xiaochen Ding. ${ }^{凶}$ email: bingliu17@126.com; tangfuchou@pku.edu.cn; rainyblue_1999@126.com
} 
334

vasculature in mammals, when instead branching morphogenesis is generally acknowledged to take place..$^{3,4,6,10-12}$

Recently, single-cell RNA sequencing (scRNA-seq) has been applied to investigate the heterogeneity of endothelial cells in distinct tissues from adult mice..$^{13-15}$ It is proposed that VECs display different properties that correspond to distinct vascular beds ${ }^{13,14}$ and present an arterial-capillary-venous zonation and seamless continuum of transcriptional states. ${ }^{13,15}$ scRNA-seq has also been used to study gastrulation and organogenesis in mouse embryos. ${ }^{16,17}$ Nevertheless, a high-precision and genome-scale gene expression landscape of early embryonic VECs is still lacking. Knowledge of the cellular evolutions and molecular programs underlying the stepwise arteriovenous fate settling will have important implications for developing new approaches for both regenerative and therapeutic purpose. Here, we used scRNA-seq, spanning the time window for the occurrence of key vasculogenic and angiogenic events in both mouse and human embryos, ${ }^{18,19}$ together with genetic lineage tracing, to decipher the endothelial heterogeneity and lineage relationships during early vascular development.

\section{RESULTS}

\section{Transcriptomic identification of distinct VEC populations in} mid-gestational mouse embryos

We first analyzed mouse embryos from embryonic day (E) 8.0, when most vessel primordia still comprise a series of disconnected clusters of Pecam1-expressing cells, ${ }^{18,19}$ to E11.0, when the first hematopoietic stem cells emerge following endothelial-tohematopoietic transition. ${ }^{20,21}$ Immunophenotypic endothelial cells $\left(\mathrm{CD} 45^{-} \mathrm{CD} 31^{+} \mathrm{CD} 144^{+}\right)$were collected from different parts of embryos $^{16,18,22}$ (Fig. 1a; Supplementary information, Fig. S1a, b and Table S1). Well-based scRNA-seq ${ }^{23}$ was performed on a total of 2213 cells (Supplementary information, Fig. S1c); $93.4 \%$ of these cells passed rigorous quality control checks with no batch effect detected (Supplementary information, Fig. S1d, e). Transcriptomically, VECs were readily distinguished from hematopoietic cells that exhibited considerable expression of Itga2b and Gata1 although they possessed an endothelial immunophenotype $22,24,25$ (Supplementary information, Fig. S2a, b). Hematopoietic cells, together with allantoic VECs that were characterized by the expression of Hoxa10 and Hoxa11 and sampled from E8.0-E8.5, ${ }^{26}$ were excluded from the subsequent analyses as they were outside the scope of this work (Supplementary information, Fig. S2a, b and Table S1).

The anatomical distribution of VECs allowed us to assign identities to the two major VEC populations as the embryo proper VEC group and the yolk sac VEC group (Fig. 1b, c; Supplementary information, Fig. S2c-g and Tables S1, S2). Genes that were highly expressed in the embryo proper VECs compared with their expression in the yolk sac VECs were mainly related to neurogenesis and artery development (Supplementary information, Fig. S2h and Table S2), consistent with the notion that VECs and neurons adopt various common mechanisms to control the patterning of vascular and neuronal networks during development. ${ }^{2}$ On the other hand, genes overrepresented in the yolk sac VEC group were predominantly associated with membrane activities, such as cell adhesion and transport (Supplementary information, Fig. S2e-h and Table S2). These findings indicated that embryonic VECs shared features associated with the biological processes of their neighboring cells, suggesting the existence of parenchymal gene signatures during the early development of VECs. Thus, from a transcriptomics point of view, the difference between intra- and extraembryonic locations was more pronounced than that between different developmental stages when discriminating between embryonic VECs (Fig. 1b, c; Supplementary information, Fig. S2e-h and Tables S1, S2).
The transcriptomically defined embryo proper VECs were further divided into eight clusters (EP0-EP7), the characteristics of which were inferred by their spatiotemporal distribution and the expression of key genes ${ }^{10,27-36}$ (Fig. 1b-e; Supplementary information, Fig. S2i-k and Tables S1, S3). EP0 mainly contained cells from E8.0 embryos, indicative of its features of primordial VECs (Fig. 1b-e). Although these earliest VECs may originate independently, their molecular characteristics were similar. Using the known arterial and venous genes, we scored the arteriovenous features of all the VEC populations (Fig. 1f, g). Hematopoietic cells were used as a negative control as they were expected to lack both arterial and venous features ${ }^{36}$ (Fig. 1f). None of the populations showed both apparent arterial and venous features (Fig. 1f), in line with the notion that arterial and venous fates are mutually exclusive. ${ }^{37}$ EP1 and EP6 manifested different degrees of venous characteristics, whereas four clusters (EP2, EP3, EP5, and EP7) displayed arterial characteristics to different degrees (Fig. 1f, g). Importantly, the clusters of embryo proper VECs could be readily distinguished in the specifically reconstituted Uniform Manifold Approximation and Projection (UMAP) by only using the genes included in the Gene Ontogeny (GO) term 'artery development' (Fig. 1h). We also evaluated the arteriovenous manifestations of yolk sac VECs, and found that arterial- or venousfeatured cells were much fewer than in embryo proper VECs at all the comparable developmental stages (Supplementary information, Fig. S2I). The further yielded three clusters of yolk sac VECs were mainly related to their developmental stages rather than arteriovenous features (Fig. 1c, f, g; Supplementary information, Fig. S2m, n). Therefore, arteriovenous characteristics made a considerable contribution to the identities of distinct VEC populations in the embryo proper but not yolk sac. We also validated the higher expression of endothelial CD36 and Neurl3 $3^{36}$ in the yolk sac of late stage (E9.5-E10.0) than in early stage (E8.5), in accordance with the transcriptomic finding (Supplementary information, Fig. S2o, p).

To determine whether the VEC populations we had identified could be readily recognized using other sequencing strategies, we performed droplet-based (10x Genomics) scRNA-seq. A total of 10,465 immunophenotypic endothelial cells from 44 embryos at E9.0-E10.0 passed rigorous quality control measures and no batch effect was detected (Supplementary information, Fig. S3a-c and Table S4). Unsupervised clustering yielded eight clusters (Supplementary information, Fig. S3d and Table S4). Of these, five clusters, which comprised the majority of the sampled cells (96.9\%), exhibited early or arteriovenous VEC characteristics (Supplementary information, Fig. S3d, e). The other three clusters were less relevant and were thus excluded from the subsequent analyses. One of these clusters was a Gata4-expressing population, which likely involved cardiac $\left(\right.$ Hand $^{+}$) and liver (Oit $3^{+}$) VECs, ${ }^{13,17,38}$ presumably due to accidental incorporation during the dissection of embryos, as the heart and visceral bud should be prospectively excluded before sampling (Supplementary information, Fig. S3d-g). The features of the five major clusters corresponded closely with the clusters identified by the well-based scRNA-seq, among which $\mathrm{Vwa}^{+}$VEC population was transcriptomically similar to EP7, with both expressing a set of arterial genes along with several specific genes, such as Vwa1 and Nid2 (Fig. 1e; Supplementary information, Fig. S3e, h). Principal Component Analysis (PCA) recapitulated the result obtained via UMAP, with the top genes of the $\mathrm{PC} 1$ axis including several known arterial markers, validating the arterial properties of the arterial VEC and $\mathrm{Vwa}^{+}$VEC clusters (Supplementary information, Fig. S3h, i). Taken together, our findings showed that identification of the principal VEC populations was reproducible using different sequencing strategies. However, given the relatively limited resolution and lack of spatiotemporal information resulting from the dropletbased scRNA-seq, we returned to the well-based scRNA-seq data for the subsequent analyses. 
a

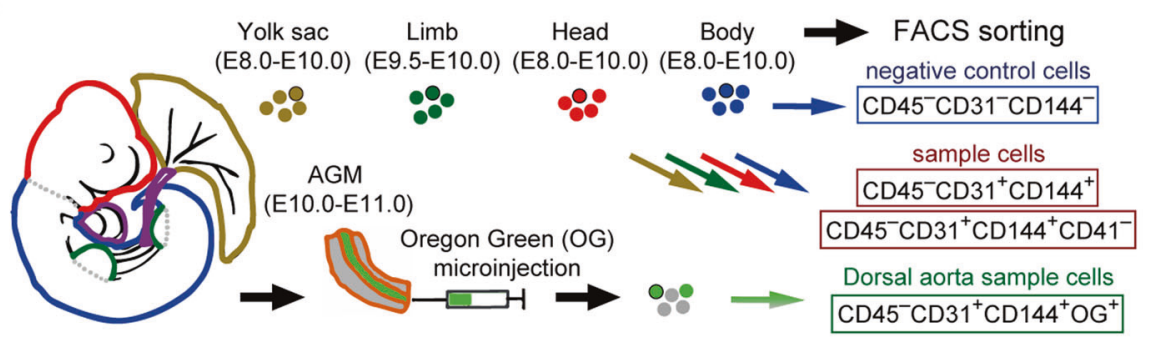

b

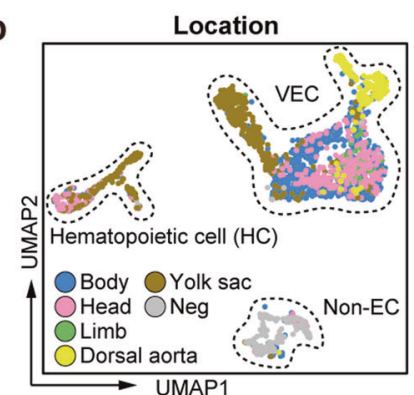

C

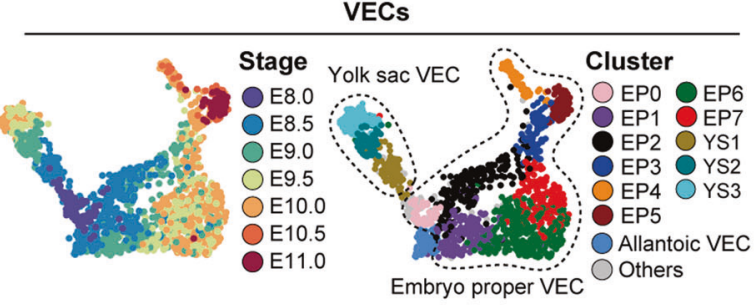

d

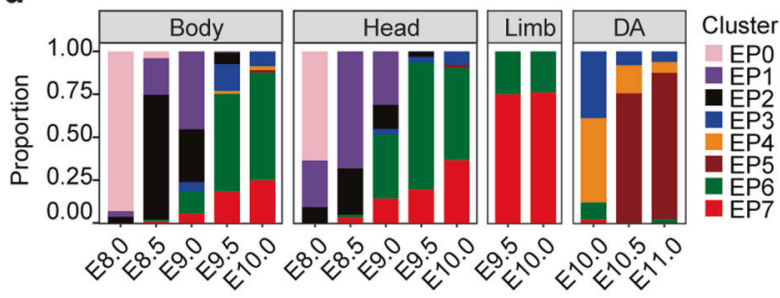

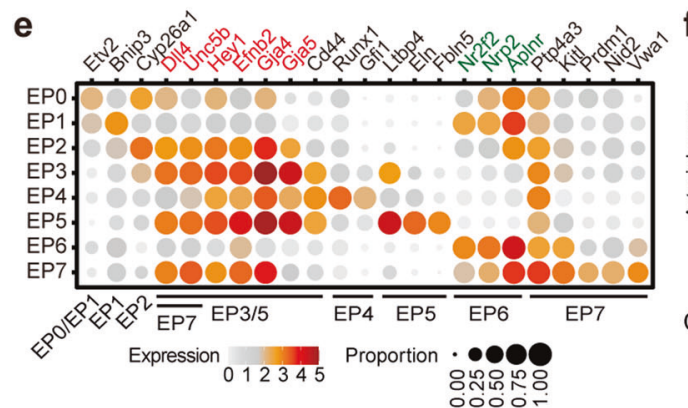

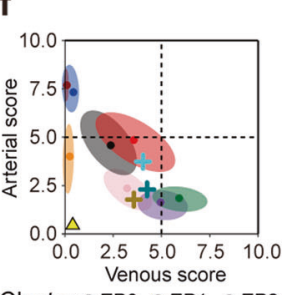

Cluster OEPO OEP1 EP2 - EP3 OEP4 ๑EP5 - EP6 $\odot E P 7+Y S 1$ g

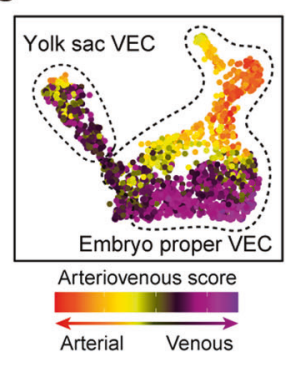

h

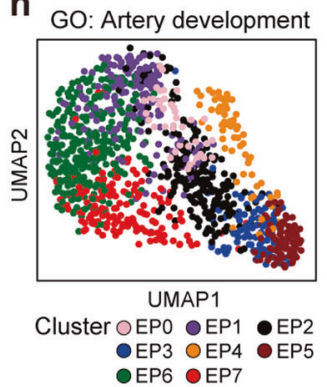

Fig. 1 Transcriptomic identification of diverse VEC populations in the mid-gestational mouse embryos. a Schematic illustration of strategies used for embryo dissection and cell preparation for the subsequent scRNA-seq. Heart, visceral bud, and umbilical and vitelline vessels outside the embryo proper (purple) were excluded. b, c UMAP plots showing sampling locations of all sequenced cells (b) and stages (c) and further yielded twelve clusters (c) of all VECs. The main areas of three subtypes of cells (b), eight clusters from embryo proper (c) and three clusters from yolk sac (c) are indicated as closed dashed curves. d Bar graph showing the proportion of each VEC cluster at the corresponding locations and stages. DA, dorsal aorta. e Dot plot showing the average expression levels and expression proportions of key feature genes distinguishing eight embryo proper VEC clusters. The size of the dot represents the proportion of cells expressing the indicated gene within a cluster, and the color indicates the average expression level of cells within a cluster. Genes in red font indicate the known arterial genes, and those in green font indicate known venous genes. The clusters to which each feature gene belongs are shown at the bottom. Note that embryo proper VEC clusters showed a biased distribution of known arteriovenous markers expect for EPO. f Scatterplot showing the average arteriovenous scores of the cells in each cluster. Main distribution ranges of arteriovenous scores in each of the eight embryo proper VEC clusters are also indicated as an oval shape. g UMAP plots showing arterial/venous scores of the individual cells in VEC clusters. The main areas of embryo proper VECs and yolk sac VECs are indicated as closed dashed curves. $\mathbf{h}$ UMAP plot with cells colored by embryo proper VEC clusters. The UMAP coordinates were determined by using 107 genes in the GO term of artery development (GO: 0060840).

\section{Spatiotemporal localization of distinct embryonic VEC populations}

To delineate the exact localization of each VEC cluster in the embryo proper, we specifically generated two pan-arterial VEC reporter mouse lines, Unc5 $b^{\text {tdTomato }}$ and DII4tdTomato, using a CRISPR/Cas9-mediated gene knockin strategy, and both expression was further validated on the postnatal retina ${ }^{39-41}$ (Fig. 2a; Supplementary information, Fig. S4a). We then developed a set of marker combinations to delineate the exact localization of principal VEC populations identified here (Supplementary information, Fig. S4b). EP1 and EP2, the two main clusters identified at E8.5 lacked Kitl expression. Anatomically, EP2 was located exclusively at major arteries, including the dorsal aortae and internal carotid arteries at E8.5, and was thus recognized as early arterial VECs (Fig. 1d, e; Supplementary information, Fig. S4b, c). In comparison, EP1 showed a vascular plexus distribution and was designated as early plexus VECs (Supplementary information, Fig. S4b, c). EP3 and EP5 were localized at dorsal aortas and their first-order side branches at E9.5-E10.5 (Fig. 2b, c; Supplementary information, Fig. S4d-f), with the relatively high Ltbp4 ${ }^{+}$signals at later stage indicative of EP5 characteristics (Supplementary information, Fig. S5a). Thus, the development from EP2 to EP3 and EP5 represented the gradual cellular progression of major artery structures.

EP6 mainly came from E9.5-E10.0 and was characterized by the expression of the venous VEC marker Nrp2 in addition to Kitl, but not arterial VEC markers (Fig. 1d, e; Supplementary information, Fig. S4b). EP6 was localized at known venous structures, such as the anterior cardinal veins, and also abundantly present in the nearby capillary plexus (Fig. 2b, c; Supplementary information, Fig. S4d-f). It was thus identified as vein \& venous plexus VECs. Distributed at the same developmental stages as EP6, EP7 expressed several known arterial genes in addition to Kitl, such as DII4, Unc5b, Hey1, Efnb2, and Gja4, but a low level of major artery genes, such as Gja5 and CD44, and venous VEC genes, such as Nrp2 and Nr2f2 (Fig. 1d, e; Supplementary information, Fig. S4b). 


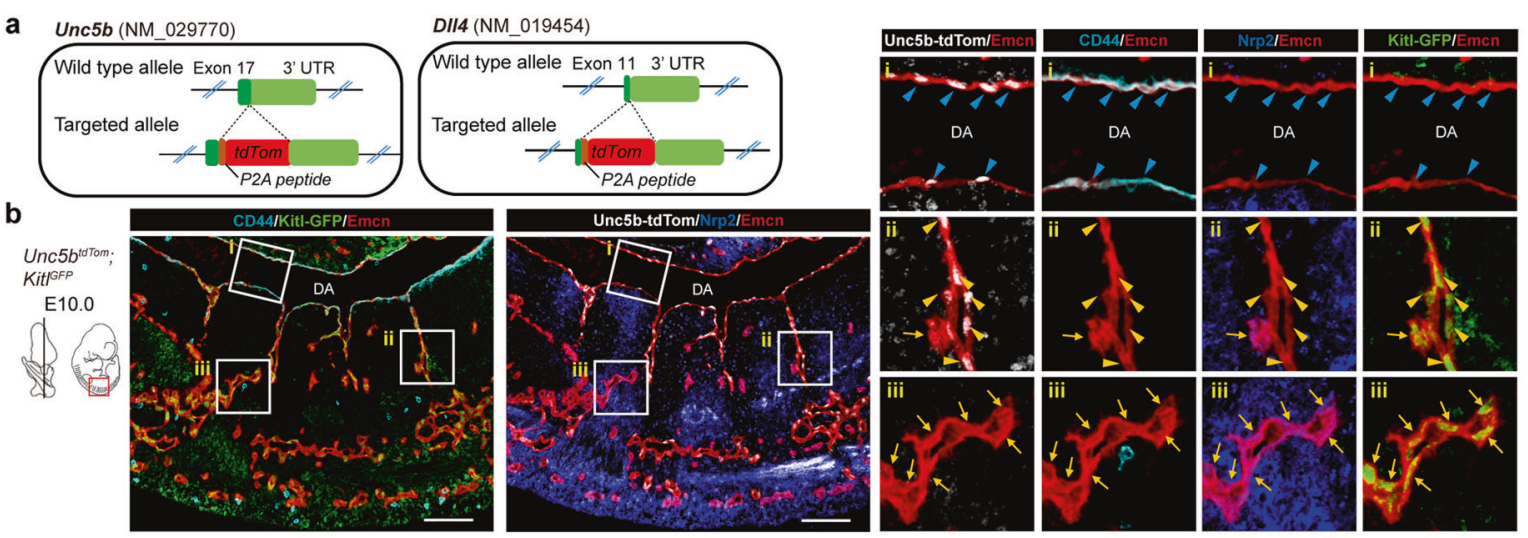

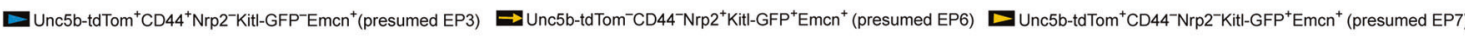
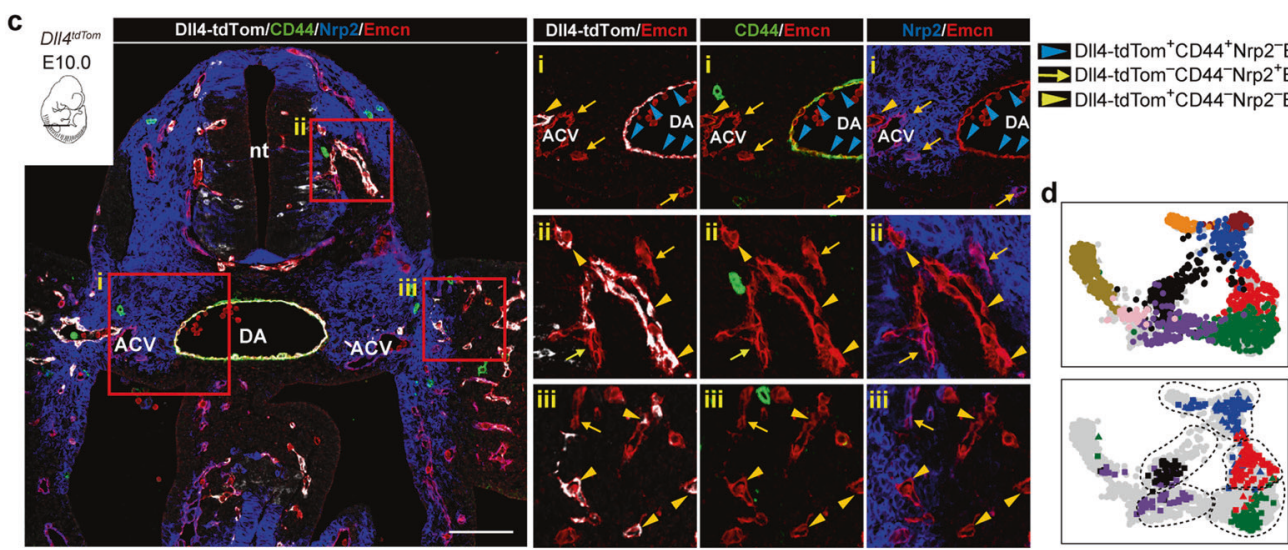

\section{$\mathrm{Cn}^{+}$(presumed EP3)} . 
a
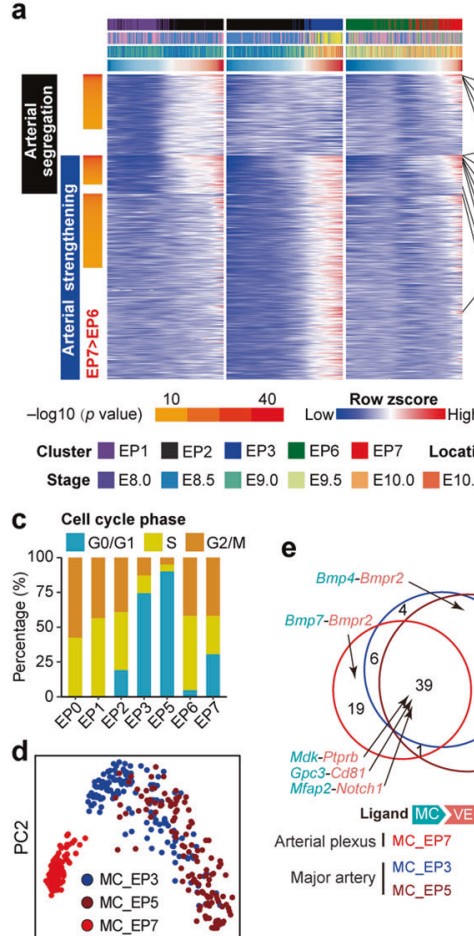

$M C-E P 7$

Major artery $\mid \begin{array}{ll}M C_{2} \text { EP3 } 3 & \text { EP3 } \\ \text { MCEP5 } & \text { EP5 }\end{array}$
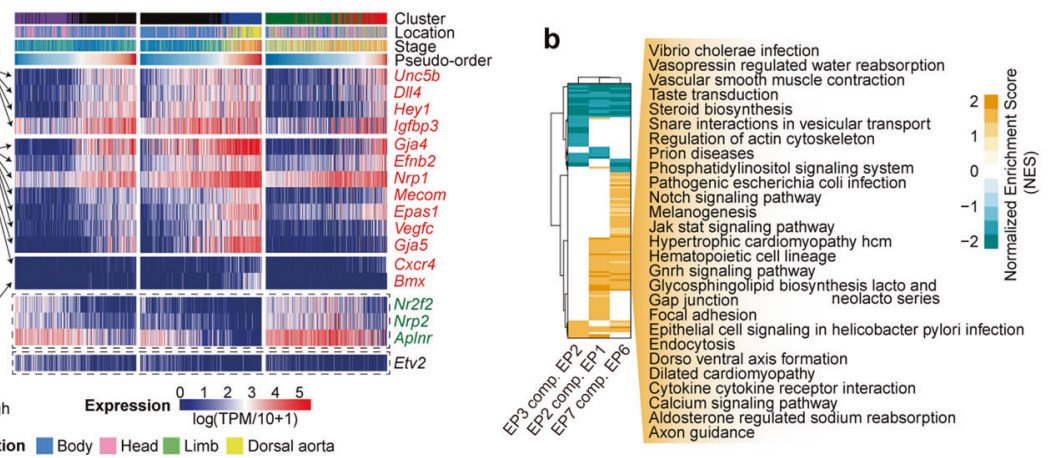
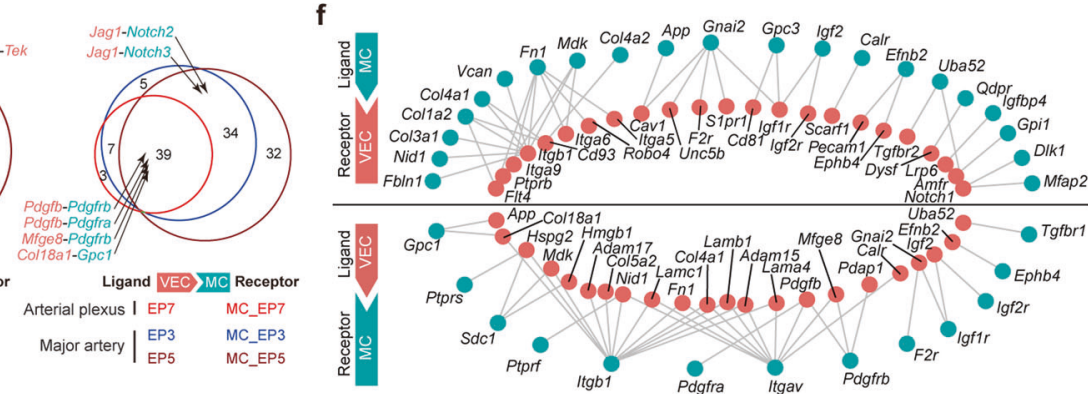

gand IMC NEC Recep

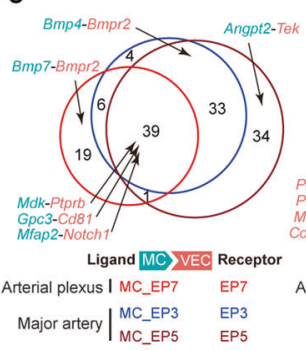

Fig. 3 Molecular evolution and predicted VEC-mural cell interactions of different arterial vasculatures in the embryo proper. a Heatmaps showing the dynamic relative expression levels of pattern genes in EP1, EP2 and EP3 with the corresponding relative expression levels in EP6 and EP7 (smoothed over 20 adjacent cells). Cells are ordered by pseudo-order axes inferred in Supplementary information, Fig. S6a. Genes are ordered firstly by pattern categories and then by $P$ values of comparison between EP6 and EP7. Expression levels of selected arterial genes from the pattern genes (red font), known venous genes (green font), and the primordial VEC maker Etv2 are shown on the right. b Heatmap showing the normalized enrichment scores (NES) of KEGG pathways by GSEA analysis between each two spatially or temporally adjacent embryo proper VEC clusters. Only significantly changed pathways (FDR $<0.1$ ) in at least one pair of comparison are shown. Row and column dendrogram are generated by using hierarchical clustering of significance indicators (1, significantly up-regulated; 0 , not significantly changed; -1 , significantly down-regulated between the indicated pairs) with Ward's linkage algorithm and Euclidean distance measure. Shared up-regulated pathways by two types of arterial VEC segregation (EP2 compared with EP1 and EP7 compared with EP6) are noted on the right. c Bar charts showing the constitutions of cells with different cell cycle phases in the indicated embryo proper VEC clusters based on molecular signatures in the scRNA-seq data. d PCA of mural cell populations. Mural cells with different derivations are shown in different colors. Note that MC_EP7 was separated from the other two mural cell populations in the dimension reduction maps, indicating their distinct molecular features. MC, mural cells. e Venn diagrams showing the numbers of shared and distinct heterologous ligand-receptor pairs between different arterial VEC populations and their corresponding mural cell populations. Representative ligand-receptor pairs are indicated. MC, mural cells. f Networks of shared heterologous ligand-receptor pairs among three arterial vasculatures. The panel above shows gene network with MC as ligand and VEC as receptor, and the panel below shows gene network with VEC as ligand and MC as receptor. MC, mural cells.

Furthermore, we isolated different endothelial populations from both DII4 $4^{\text {tdTomato }}$ and Unc5 $b^{\text {tdTomato }}$ reporter mouse lines using the same marker combinations as those used for immunostaining, and performed scRNA-seq (Supplementary information, Fig. S5c and Table S5). Importantly, when projected onto the original UMAP, the newly sequenced VEC populations clustered together with the cell populations that they were presumed to be (Fig. $2 d$, e). We also validated previously unknown markers of two arterialfeatured clusters with the use of RNAscope. As a marker gene of early arterial VECS (EP2) (Fig. 1e; Supplementary information, Fig. S2k), endothelial Cyp26a1 expression was confined to the aortae at E8.5 and was absent at E10.0 (Supplementary information, Fig. S5d). Ptp4a3 was transcriptionally activated in arterial plexus VECs (EP7) (Fig. 1e), which, anatomically, showed a predominantly endothelial expression pattern. The location was restricted to some small vessels, including intersegmental branches from the dorsal aorta, in accordance with that of EP7, identified by the known arterial markers Dll4 or Unc5b (Supplementary information, Fig. S5d).

Taken together, two types of arterial VECs, namely the major artery VECs (EP3 and EP5) and arterial plexus VECs (EP7) could be readily discriminated, whereas the morphologically well-defined major veins and venous plexus were largely indistinguishable on a molecular basis. The asymmetric arteriovenous characteristics are different to those seen in adults, where VECs display different properties that correspond to distinct vascular beds ${ }^{13,15}$ (Fig. 2f).

Molecular characteristics and mural cell properties of different arterial vasculatures in the embryo proper

We next investigated changes in gene expression across spatially or temporally continuous VEC populations. The pseudo-order within EP1 and EP2 inferred a continuum of arterial-venous identity on the initially established circulatory loop, whereas that within EP2 and EP3 represented the molecular changes occurring in major arteries during their development (Fig. 3a; Supplementary information, Fig. S6a and Table S3). We revealed three main expression patterns which respectively represented changes in segregation into the first arterial fate and/or in the subsequent strengthening of arterial features (Fig. 3a; Supplementary information, Table S3), and found that the expression of most arterial segregation-related genes was also elevated in EP7 compared with their expression in EP6, with many acknowledged arterial 
338

markers highly ranked (Fig. 3a; Supplementary information, Table S3). The similarity between these two types of arterial fate segregation, namely the initial major artery fate settling (EP2 as compared with EP1) and arterial fate segregation in the vascular plexus (EP7 as compared with EP6) was further supported by their considerable sharing of significantly enriched signaling pathways (Fig. 3b; Supplementary information, Table S3), top differentially expressed genes and transcription factors, and enriched terms (Supplementary information, Fig. S6b-g). Cell cycle analysis of distinct VEC populations demonstrated an increase in quiescent status accompanied by arterial feature segregation, strengthening, and maturation, including when EP7 was compared with EP6 (Fig. 3c). This was in accordance with previous findings showing the enabling role played by cell cycle suppression in arterial differentiation. ${ }^{10,42,43}$ The different proliferation status of distinct VEC populations was also validated by flow cytometric evaluation of Ki67 expression and BrdU incorporation with subsequent immunostaining (Supplementary information, Fig. S6h-j).

Different arterial VEC populations showed distinct mural cell coverage status around E10. PDGFR $\beta$-expressing mural cells were distributed among all the arterial vasculatures that we identified (major arteries and the arterial plexus), whereas a-SMA-expressing mural cells were restricted to the major arteries (Supplementary information, Fig. S7a, b). We further isolated PDGFR $\beta$-expressing mural cells around the dorsal aorta at E9.5 and E10.5 and in limb buds at $\mathrm{E} 9.5{ }^{44}$ corresponding to three different arterial populations including two major artery VEC populations EP3 and EP5 and arterial plexus VECS EP7, respectively, and performed scRNA-seq (Fig. 3d; Supplementary information, Fig. S7a-c and Table S6). Of note, most of the heterologous ligand-receptor pairs in the arterial plexus were shared with those in the major arteries, implying largely similar mechanisms underlying the arterial stabilization mediated by mural cell-VEC interactions, irrespective of the vascular beds (Fig. 3e, f; Supplementary information, Fig. S7d, e and Table S6). More potential interactions were found in major arteries than in the arterial plexus, with E10.5 having the highest number, suggesting enhanced mural cell-VEC interactions during artery maturation (Fig. 3e, f; Supplementary information, Fig. S7e and Table S6).

\section{Transcriptomic identification of two types of arterial VEC populations in human embryos}

To determine whether our finding with respect to the two types of embryonic arterial VECs is conserved among mammals, scRNA-seq was performed with immunophenotypic endothelial cells derived from different intra-embryonic sites of totally five human embryos that were spatiotemporally comparable to mouse embryos used in this study ${ }^{45,46}$ (Supplementary information, Fig. S8a, b and Table S7). A total of 835 of $966(86.4 \%)$ sampled cells passed a strict quality control assessment, and the batch effect and cell cycle effect were regressed out (Supplementary information, Fig. S8b, c and Table S7). Similar to the result of 10x Genomics scRNA-seq of mouse VECs (Supplementary information, Fig. S3d-g), a GATA4 ${ }^{+} \mathrm{HAND2}^{+}$and a GATA4 $4^{+} \mathrm{OIT3}^{+}$cluster, possibly corresponding to cardiac and liver endothelial cells, respectively, were also identified ${ }^{38,47}$ (Supplementary information, Fig. S8d, e). These two minor clusters, together with a hematopoietic cell cluster featured by the expression of erythroid cell marker GYPA and the presumed hemogenic endothelial cells characterized by RUNX1 expression, were excluded; the two remaining vascular VEC clusters of interest were retained for further analyses. ${ }^{46}$

Human embryonic VECs were combined with mouse embryo proper VECs identified by the same sequencing strategy for the integration and comparison analysis (Figs. 1b, c, 4a; Supplementary information, Fig. S8d, e and Table S7). The assignment accuracy was further confirmed by correlation analysis in Mutual Nearest Neighbors (MNN) Space (Supplementary information,
Fig. S8f). Some human VECs were clearly assigned to the two types of arterial-featured VECs, namely major arterial VECs EP3 and EP5, which were together renamed as AEC1 to simplify the annotation, and arterial plexus VECS EP7, which were renamed hereafter as AEC2 (Fig. 4a-C; Supplementary information, Fig. S8f, $g$ and Table S7). Both human AEC populations showed a higher proportion of cells in a quiescent status compared with the venous-featured VEC population EP6 (renamed hereafter as VeEC), with $A E C 1$ being the most quiescent (Supplementary information, Fig. S8h). In addition, enriched terms of the highly expressed genes in human $A E C 2$ but not AEC1 showed characteristics that suggested a propensity toward cell migration and motility (Fig. 4d).

We next determined conserved arteriovenous feature genes in these two species during vascular development. A total of 68 and 10 conserved genes were screened out for major arterial VECs (AEC1) and arterial plexus VECs (AEC2), respectively (Fig. 4e; Supplementary information, Fig. S8i and Table S7). On the other hand, only four genes were screened out as conserved genes for the embryonic venous-featured VEC population, including the known venous markers NR2F2, NRP2, and APLNR (Fig. 4e). We also made an effort to identify conserved arterial genes, which were defined as those highly expressed in both AEC populations when respectively compared with the corresponding venous VEC population. A total of 20 conserved arterial genes were identified, with the 9 most conserved ones being well-known arterial genes, including three transcription factors, MECOM, HEY1, and EPAS1, as well as UNC5B and DLL4 (Fig. 4f; Supplementary information, Fig. S8j), the homologs of which in mouse were used as panarterial markers for the in situ validation in this study (Figs. 1e and $2 b$, c; Supplementary information, Figs. S4c-f and S5b).

Finally, an integrated single-cell intra-embryonic VEC map within the time window of our interest was constructed, which included the datasets of both mouse and human embryonic VECs developed here, using both well-based and droplet-based sequencing strategies. This will act as a resource to facilitate future studies of early vascular development in mammals (Fig. 4g; Supplementary information, Fig. S8k and Table S7).

Evolutionarily conserved developmental paths of two types of arterial VECs predicted by trajectory analyses

Mpath $^{48}$ predicted two developmental paths of VECs from primordial VECs (EP0). One was toward mature arterial VECs (EP5), experiencing early arterial VECs (EP2) and the subsequent maturing arterial VECs (EP3), which represented the step-by-step cellular changes of major artery VECs. The other was toward early plexus VECs (EP1) and further vein \& venous plexus VECs (EP6), with the final fate as arterial plexus VECs (EP7), reflecting the cellular evolution of the vascular plexus (Fig. 5a). Trajectory analysis by Monocle 3 at single-cell resolution ${ }^{17}$ largely recapitulated the actual temporal order of the sampled cells and showed two major directions (Fig. 5b). In general, two waves of arterial fate were sequentially segregated out. One came directly from primordial VECs, known as via vasculogenesis, and the other involved the branching of arterial plexus VECs from the venousfeatured VEC population, through angiogenesis and remodeling (Fig. 5b-d; Supplementary information, Table S1). We also performed RNA velocity analysis to predict the future state of each individual cell, based on an algorithm that was distinct from other trajectory analyses. ${ }^{49}$ The results were consistent with the findings from Mpath and Monocle 3, showing two waves of arterial differentiation, one of which was presumably derived from vein \& venous plexus VECs (EP6) (Fig. 5e). Taken together, trajectory analyses by distinct algorithms all indicated the occurrence of venous plexus arterialization (Fig. 5c; Supplementary information, Table S1).

We next explored whether our finding with respect to the development paths of two types of embryonic arterial VECs is 
a

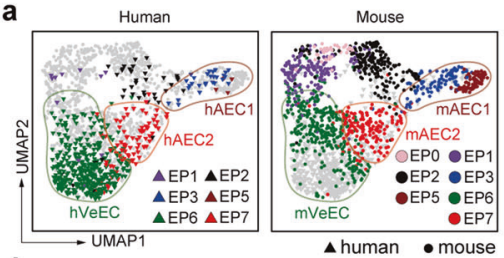

d

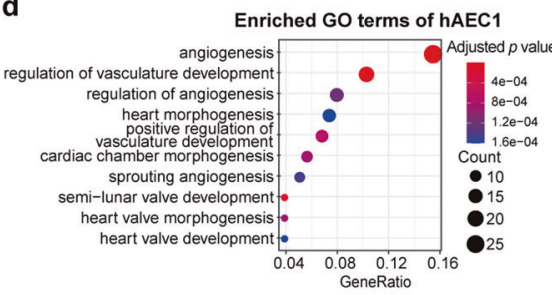

Enriched GO terms of hAEC2

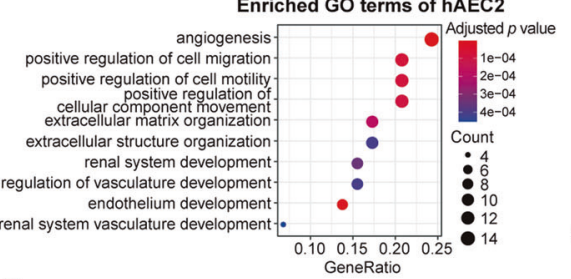

g

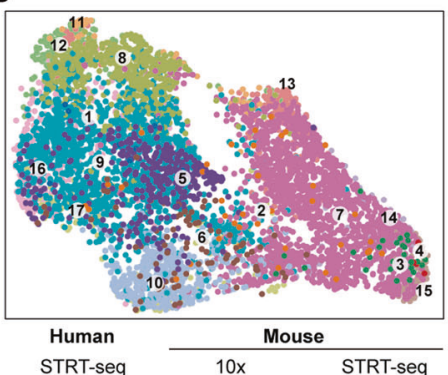

(1) EP1 (4) EP5 7 Arterial VEC 11 EPO 15 EP5

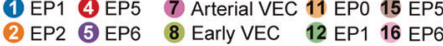

(3) EP3 6 EP7 9 Venous VEC 13 EP2 17 EP7

$10 \mathrm{~V}_{\text {wa }}{ }^{+}$VEC 14 EP3

b

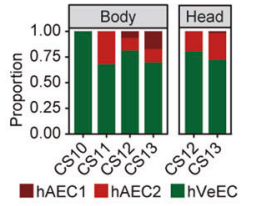

C

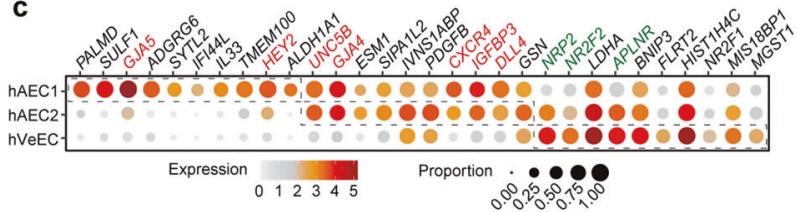

e

e
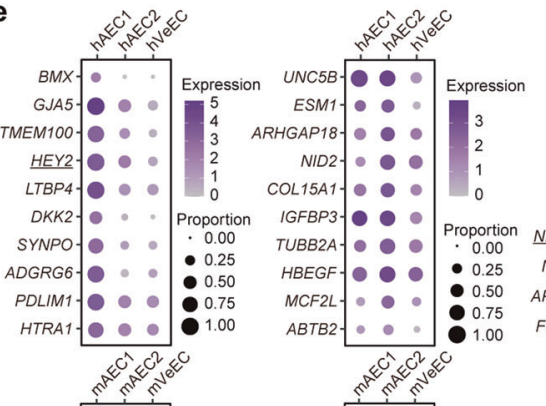

f Conserved arterial genes
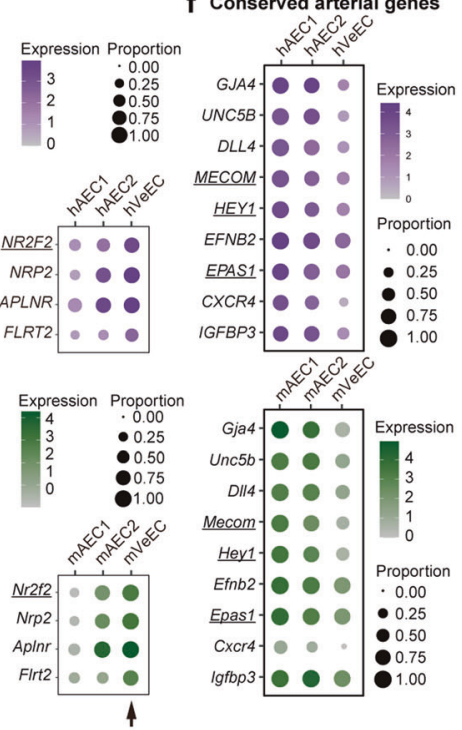

Human STRT-seq
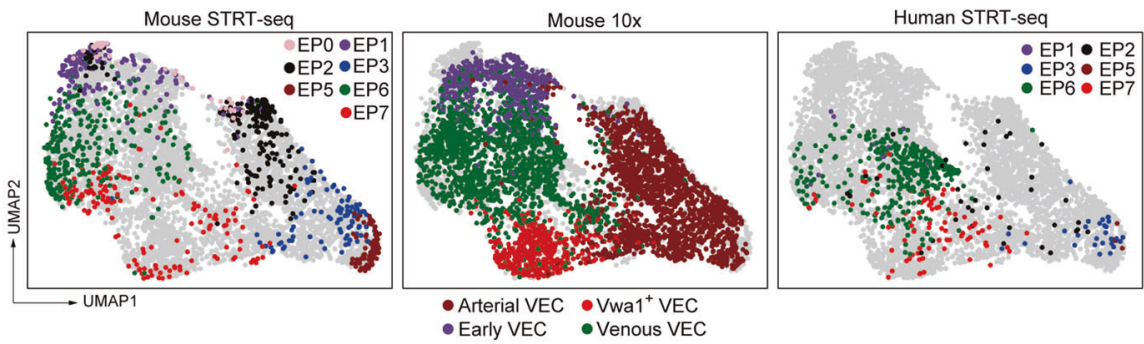

Fig. 4 Transcriptomic identification of different arterial VEC populations in human embryos. a UMAP plots showing integrated analysis of VECs from mouse and human embryos by STRT-seq. The human EP3 and EP5 combination was renamed as human arterial VEC1 (hAEC1), EP7 as hAEC2, and EP6 as human venous VEC (hVeEC), corresponding to the mouse EP3 and EP5 combination (mAEC1), EP7 (mAEC2), and EP6 ( $\mathrm{mVeEC}$ ), respectively. b Bar graph showing the proportion of each human cluster at the corresponding locations and stages. CS, Carnegie stage. c Dot plot showing the expression levels of top ten marker genes for each of the three indicated human VEC populations. Note that hAEC1 and hAEC2 shared several classical arterial genes, whereas hAEC1 specifically overrepresented GJA5 and HEY2. Known arterial and venous genes are indicated in red and green, respectively. $\mathbf{d}$ The top ten enriched GO biological process terms of hAEC1 (upper) and hAEC2 (lower). Dot color indicates the statistical significance of the enrichment and dot size represents the number of genes annotated to each term. e, $\mathbf{f}$ Dot plots showing the average expression level and expression percentage of the top ten (all included if less than ten) conserved genes for each of the three types of arteriovenous VECs as indicated by arrows (e) and those of the nine conserved arterial genes, when defined as significantly overrepresented in all the four comparisons (two arterial clusters respectively compared to the corresponding venous cluster in both human and mouse) (f). Size of the dot represents the percentage of cells expressing the indicated gene within a cluster, and color indicates the average expression level of the gene within a cluster. Genes are listed in ascending order of Fisher's combined $P$ value from independent $P$ values of human and mouse datasets. Genes encoding transcription factors are underlined. g UMAP plots showing integrated analysis of three embryonic VEC-related datasets from human and mouse embryos and sequenced by using STRT-seq and 10x Genomics methods.

conserved among mammals. Trajectory analysis within the integrated dataset of human and mouse by Monocle 3 also identified two predominant paths from primordial VECs (Fig. 5f). The gradual changes in arteriovenous features along the respective paths of these two waves of arterial VEC development were similar between human and mouse (Fig. 5g; Supplementary information, Fig. S8I). Specifically, mitotic nuclear division-related genes were enriched in the gene set with an expression pattern that showed gradual down-regulation along major artery VEC development in both human and mouse (Supplementary information, Fig. S8I). Taken together, it was basically conserved in mid-gestational vascular development between human and mouse regarding the two major types of arterial VEC populations and their developmental paths.

\section{Widespread capillary arterialization from venous-featured plexus during early expansion of intra-embryonic vasculatures}

Finally, we explored whether arterial plexus VECs arise from arterialization of venous plexus VECs, as deduced from the bioinformatics analysis. We specifically chose the canonical venous marker $\mathrm{Nr} 2 \mathrm{f} 2$ and generated a $\mathrm{Nr} 2 \mathrm{f2}{ }^{\mathrm{CrexER}}$ mouse line which allows inducible genetic lineage tracing (Fig. 6a). First, we checked the expression of CrexER by ER immunostaining from 

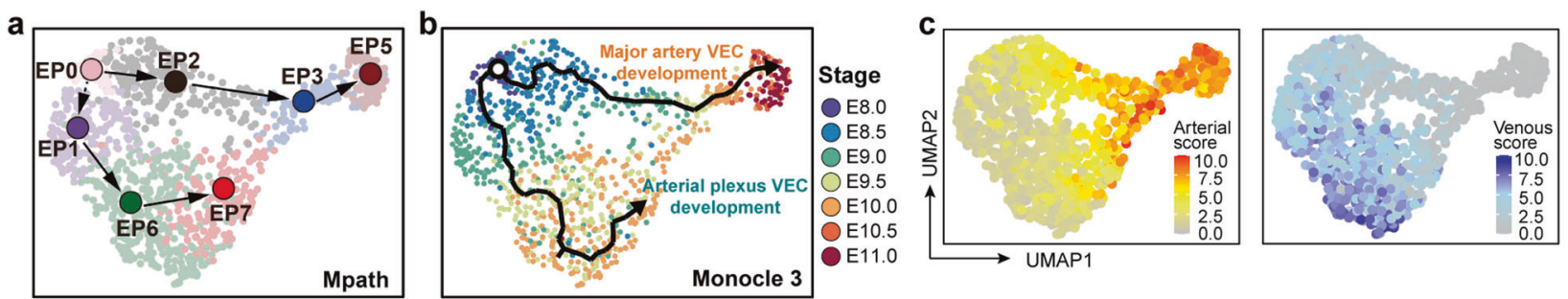

d
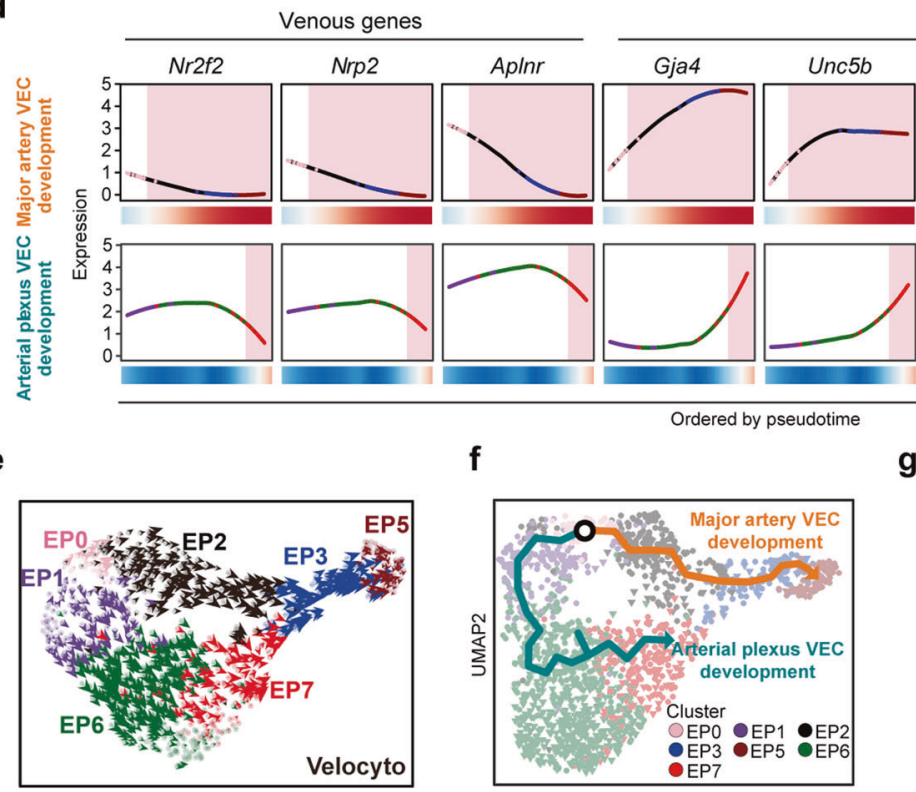

Arterial genes

Dll4 Mecom
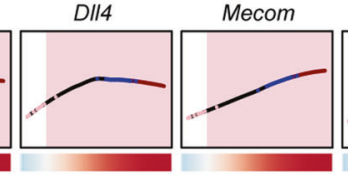
Hey1
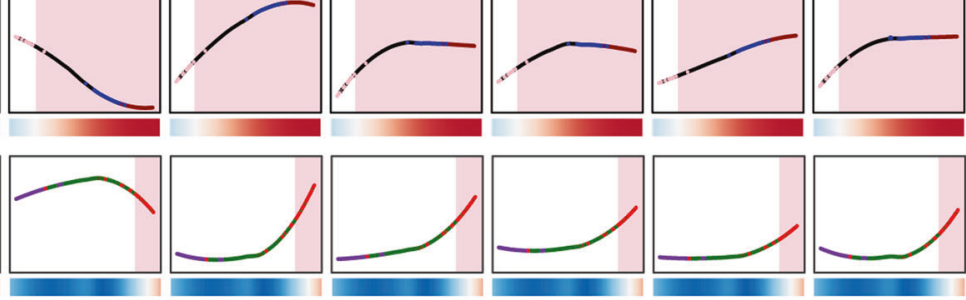

Cluster Arteriovenous EPO score -EP1 - EP2

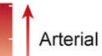

Ordered by pseudotime
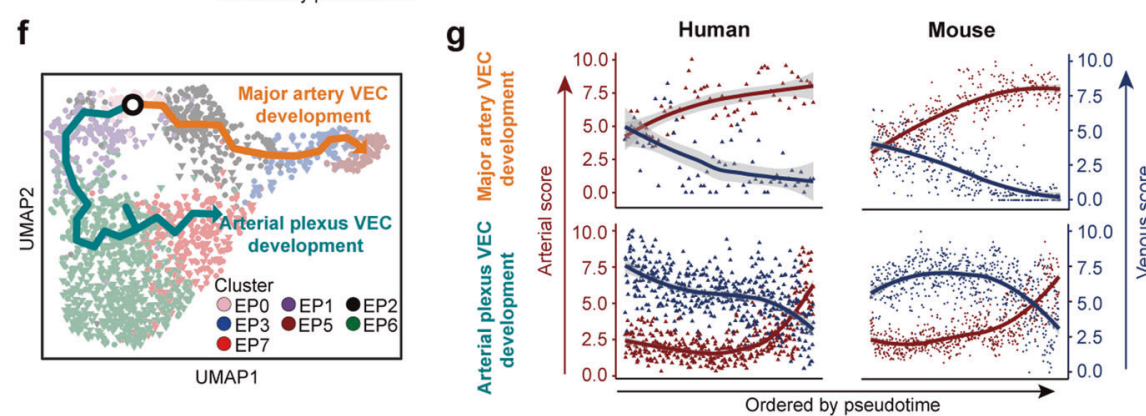

Fig. 5 Computational analysis predicted the basically conserved development paths of two types of arterial VECs between human and mouse. a Developmental trajectory among embryo proper VEC clusters inferred by Mpath. The directional arrows are inferred by Mpath and sampling stages. b Trajectory of cells in embryo proper VEC clusters inferred by monocle 3 showed two major directions mapped with sampling stages, which largely recapitulated the actual temporal order of the sampled cells. c UMAP plots showing arterial (left) and venous (right) scores of the individual cells in the embryo proper VEC group. $\mathbf{d}$ Loess regression-smoothened expression of the indicated venous and arterial marker genes along two distinct arterial VEC development paths inferred by Monocle 3. Smoothened arteriovenous scores are also shown at the bottom. Red shading, arterial-featured VEC populations. e Velocity vector field displayed on the UMAP plot at single-cell level, with each arrow colored by cluster showing the movement direction and speed of each individual cell, indicating two waves of arterial differentiation, one of which was presumably derived from vein \& venous plexus VECs (EP6). f UMAP plot showing the trajectory inferred by Monocle 3, which identified two predominant paths from primordial VECs. g Scatter plots showing arterial (red) and venous (blue) scores of the cells along major artery VEC development (upper) and artery plexus VEC development (lower) paths, with loess-smoothed fit curves indicated. VECs from human and mouse embryos are illustrated separately, and cells are ordered by pseudotime.

E8.0 to E9.5 and confirmed that its expression was in general consistent with that of $\mathrm{Nr} 2 \mathrm{f2}$ at the transcriptional level (Fig. 1e; Supplementary information, Fig. S9a-c). Nr2f2-CrexER was undetectable in the vasculature at E8.0, in contrast to Nrp2 that exhibited scattered expression in the aortae and ubiquitous yet inconsistent expression in other vascular primordia (Fig. 1e; Supplementary information, Fig. S9a). At later stages, Nr2f2CrexER was expressed in the anatomically recognized veins and also in the vascular plexus, showing a mutually exclusive expression pattern with that of DII4. This was similar to the performance of Nrp2 with regard to the vasculature (Supplementary information, Fig. S4c-e). These findings provided support for the identity of Nr2f2-CrexER as a venous-featured marker to label both early plexus VECs (EP1) and vein \& venous plexus VECs (EP6) but neither primordial VECs nor arterial-featured VEC populations (Fig. 1e; Supplementary information, Fig. S9b, c). We also generated a Tie2-Dre;Nr2f2-CrexER mouse model, in which the Cre-mediated ablation occurred specifically in the $\mathrm{Nr} 2 \mathrm{f2}$ expressing VECs but not in other cell types ${ }^{50}$ (Fig. 6b), to constitutively trace the fate of venous-featured VECs and achieve better vasculature visualization.
Using either Rosa-EYFP or Rosa-H2b-mCherry as the reporter of Cre recombinase, we performed genetic lineage tracing. We confirmed there was no leakiness of Nr2f2-CrexER without tamoxifen induction (Supplementary information, Fig. S10a). At E10.0, Tie2Nr2f2-Cre lineage-labeled cells were widely distributed throughout the vasculature, but were seldom detected in the aorta, similar to that traced by a single dose of tamoxifen induction at E8.5 and observed at E11.0 (Supplementary information, Fig. S10b, c). These results were in line with the previous notion and also the trajectory analysis here, indicating that the initial fates of major artery VECs and venous VECs have already been segregated by the time when VECs acquire Nr2f2 expression and become venous at around E8.5 (Fig. 5b, e). Histologically, the contribution of the Tie2Nr2f2-Cre lineage to the Unc5 $\mathrm{b}^{+}$arterial plexus VECs was evident, including those located at the branches of internal carotid arteries, the intersegmental arteries, and the limb bud arterial plexus (Fig. 6c; Supplementary information, Fig. S10d). By further scRNA-seq with the isolated Tie2Nr2f2-Cre lineage-labeled VECs, we clearly showed the arterial characteristics of most lineage-traced Unc $5 \mathrm{~b}^{+}$VECs with a loss of $\mathrm{Nr} 2 \mathrm{f2}$ expression, providing molecular validation of the conversion from 
Nr2f2 (NM_009697) Wild type allele Exon3 3' UTR

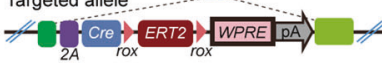

b

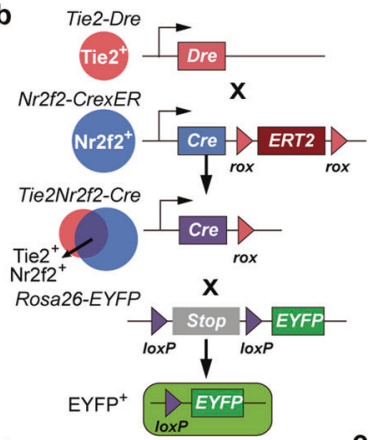

d

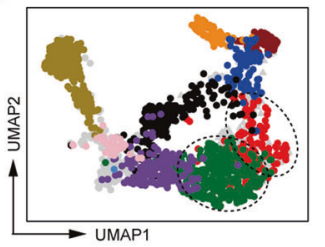

EP0 •EP1 •EP2 •EP3 | Original - EP4 •EP5 •EP6 •EP7 data Tie2Nr2f2-Cre lineage

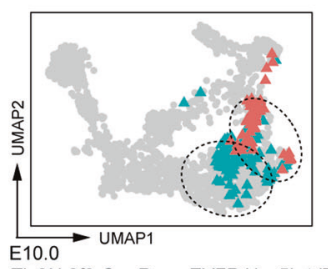

Tie2Nr2f2-Cre;Rosa-EYFP;Unc5b-tdTom $\triangle$ EYFP $^{+}$VECS $\triangle$ EYFP $^{+}$Unc5b-tdTom ${ }^{+} V E C s$ Tie2Nr2f2-Cre lineage

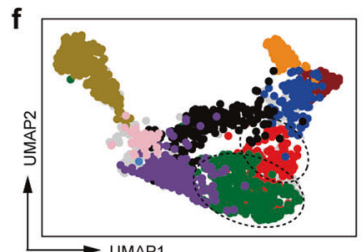

EP0 •EP1 •EP2 •EP3 |Original - EP4 $\bullet$ EP5 $\bullet$ EP6 •EP7
- Yolk sac VEC Other Nr2f2-CrexER lineage

e
C Tie2Nr2f2-Cre;Rosa-EYFP;Unc5b-tdTom E10.0
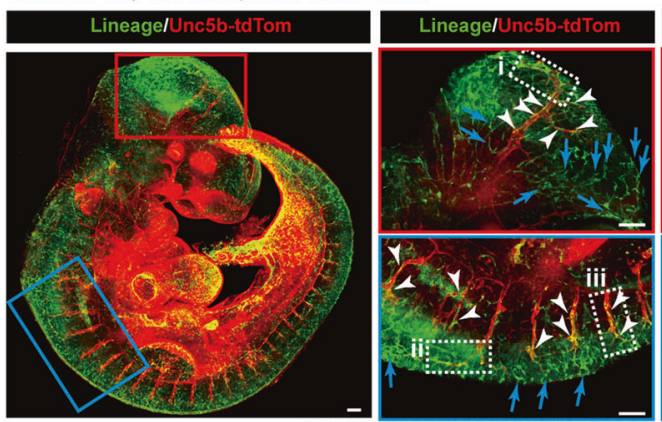

Lineage ${ }^{+}$Unc5b-tdTom ${ }^{+}$VECs $\Rightarrow$ Lineage ${ }^{+}$Unc5b-tdTom ${ }^{-}$VECs
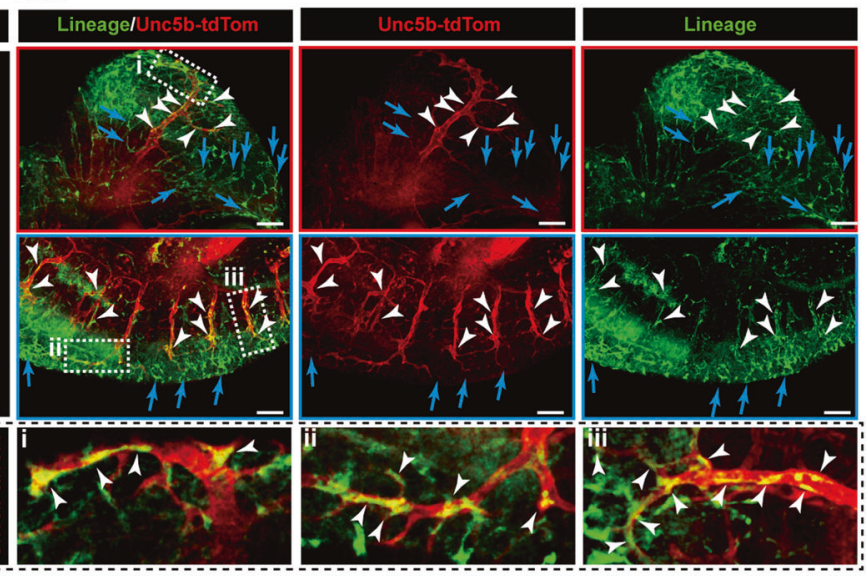

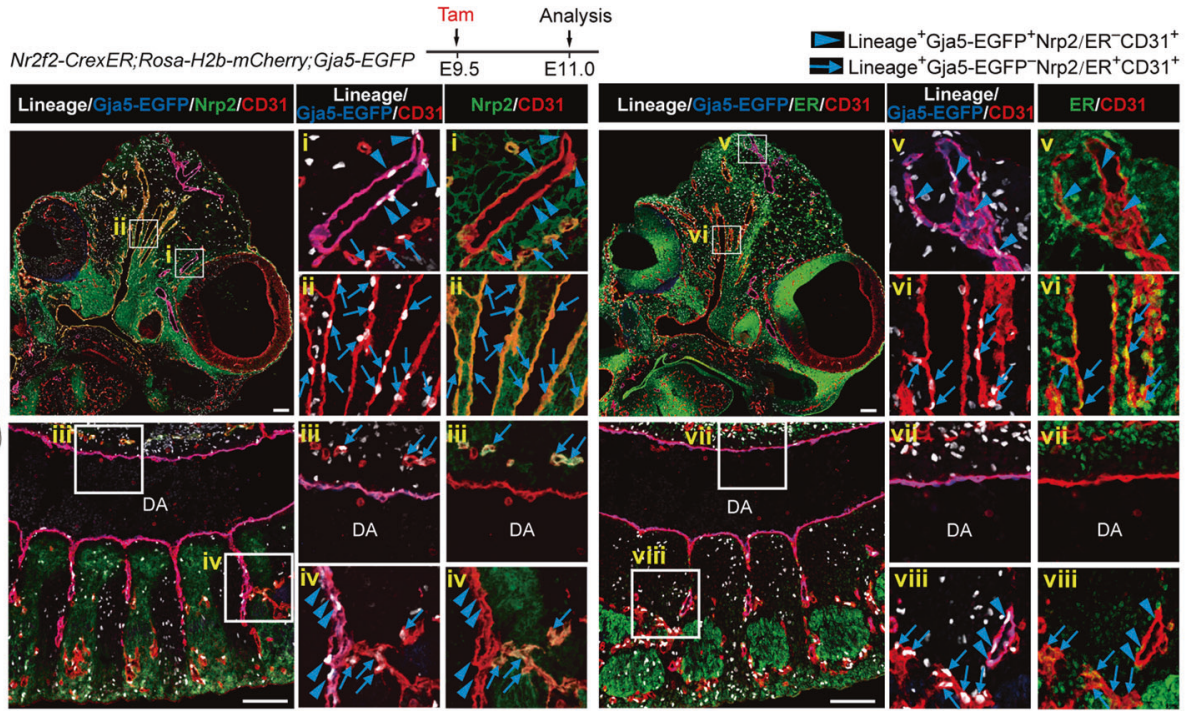

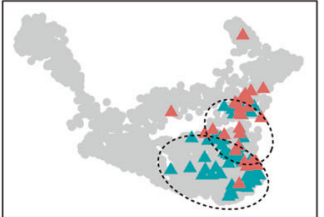

E11.0

Nr2f2-CrexER;Rosa-H2b-mCherry;Gja5-EGFP $\mathrm{H}_{2} \mathrm{~b}-\mathrm{mCherry}+\mathrm{VECs}$ b-mCherry ${ }^{+}$Gja5-EGFP ${ }^{+}$VECs

g Arteriovenous specification of early intra-embryonic vasculature

Conventional model Branching morphogenesis and/or capillary specification

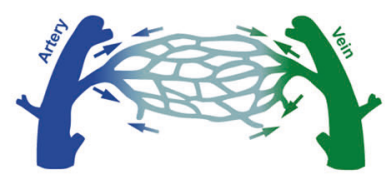

Proposed model Venous arterialization

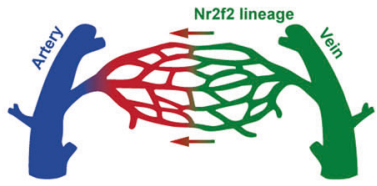

Fig. 6 Genetic lineage tracing showing the capillary arterialization from venous-featured plexus during early expansion of intraembryonic vasculatures. a Schematic model of the gene-targeting strategy for generating Nr2f2-CrexER mouse line via CRISPR/Cas9 system. b Schematic model showing the working principle for sequential Dre and Cre recombination. The final readout is Cre-loxp recombination. c Whole-mount immunostaining of E10.0 Tie2Nr2f2-Cre;Rosa-EYFP;Unc5b-tdTomato embryo with antibodies of RFP (red) and GFP (green) to detect Unc5b-tdTomato ${ }^{+}$cells and Tie2Nr2f2-Cre lineage-traced cells, respectively. Note that in addition to the Unc5b-tdTom ${ }^{-}$VECs (blue arrows), the labeling by Tie2Nr2f2-Cre lineage is also observed in the Unc5b-tdTom ${ }^{+}$arterial VECs (white arrowheads), the distribution of which includes the branches of internal carotid arteries and the intersegmental arteries. Images in red boxes, reconstructions with a total of $70 \mu \mathrm{m}$ thickness; images in blue boxes, reconstructions with a total of $100 \mu \mathrm{m}$ thickness; images in dotted boxes show inserts at high magnification. Scale bars, $100 \mu \mathrm{m}$. d UMAP plots combining the cells from our original data and E10.0 Tie2Nr2f2-Cre lineage-labeled VECs $\left(C D 41^{-} \mathrm{CD} 43^{-} \mathrm{CD} 45^{-} \mathrm{CD} 31^{+} \mathrm{CD} 144^{+}\right)$. Note that the lineage-labeled VECs are mainly clustered together with EP6 and EP7. Dashed outlines of EP6 and EP7 are indicated. e Representative immunostaining on sagittal sections of E11.0 Nr2f2-CrexER;Rosa-H2b-mCherry;Gja5-EGFP embryos after single dose of tamoxifen induction at E9.5. Note that in addition to the Gja5-EGFP ${ }^{-} \mathrm{Nrp}_{2} / \mathrm{ER}^{+} \mathrm{CD} 31^{+}$venous VECs (blue arrows), the labeling by Nr2f2-CrexER lineage is also observed in the Gja5-EGFP ${ }^{+} \mathrm{Nrp}_{2} / \mathrm{ER}^{-} \mathrm{CD} 31^{+}$artery plexus VECs (blue arrowheads), the distribution of which includes the branches of internal carotid arteries and the intersegmental arteries but not DA. The diagrams on the left indicate the positions of the sections and imaging. DA, dorsal aorta. Scale bars, $100 \mu \mathrm{m}$. f UMAP plots combining the cells from our original data and E11.0 Nr2f2-CrexER lineage-labeled VECs (CD41 $\left.{ }^{-} \mathrm{CD} 43^{-} \mathrm{CD} 45^{-} \mathrm{CD} 31^{+} \mathrm{CD} 144^{+}\right)$. Note that the lineage-labeled VECs are mainly clustered together with EP6 and EP7. Dashed outlines of EP6 and EP7 are indicated. g Diagram showing the conventional model (left) and newly proposed model (right) regarding the arteriovenous specification of early intra-embryonic vasculature. 
venous- to arterial-featured VECs (Fig. 6d; Supplementary information, Fig. S10e, $f$ and Table S5).

Single dose tamoxifen induction in the Nr2f2-CrexER;Rosareporter mouse model at E9.5 labeled mainly vein \& venous plexus VECs (EP6) but few, if any, early plexus VECs (EP1) (Fig. 1d, e). Importantly, at E11.0, $\mathrm{Gja}^{+}$and Unc5b ${ }^{+}$arterial plexus VECs but not aortic VECs were evidently lineage-traced by E9.5 induction, although the tracing efficiency varied at least in part due to the different Rosa-reporter systems (Fig. 6e; Supplementary information, Fig. S11a). Furthermore, the ER expression in the lineage-traced arterial plexus VECs was largely diminished, validating the conversion from venous- to arterial-featured VECs histologically (Fig. 6e; Supplementary information, Fig. S11a). Flow cytometric analysis further confirmed that tamoxifen treatment at E9.5 led to the labeling in both DII4 ${ }^{-}$CD $44^{-}$VECs (presumed EP6, vein \& venous plexus VECs) and DII4 ${ }^{+}$CD $44^{-}$VECs (presumed EP7, arterial plexus VECs) in multiple embryonic sites, but seldom in DII ${ }^{+} \mathrm{CD}_{4} 4^{+}$VECs in the aorta-gonad-mesonephros (AGM) region (major artery VECs) (Supplementary information, Fig. S11b). We also performed additional scRNA-seq of the Nr2f2-CrexER lineagelabeled VECs with Gja5-EGFP as an arterial indicator, and validated the unambiguous arterial identity of partial Nr2f2-CrexER lineagetraced cells when induced at E9.5 (Fig. 6f; Supplementary information, Fig. S11c, d and Table S5).

Regarding the distribution of the lineage-traced cells within the vasculature and their contribution to the arterial-featured VECS, the lineage-tracing results of those induced at E9.5, those induced at E8.5, or using the constitutive Tie2; $\mathrm{Nr} 2 \mathrm{f2}-\mathrm{Cre}$ system were similar. This indicated that the conversion to an arterial fate predominantly occurred from the population with more apparent venous characteristics (EP6) rather than directly from early plexus VECS (EP1), in line with the prediction of the trajectory analysis (Fig. 5a, b, e). Taken together, genetic lineage tracing clearly revealed early and extensive capillary arterialization from venousfeatured plexus during mouse embryonic development, involving multiple anatomical sites. This reshapes the conventional model of arteriogenesis, which predominantly involves branching morphogenesis from pre-existing arteries and symmetric remodeling with arterial specification from unspecialized capillaries during the expansion of early vasculature ${ }^{4}$ (Fig. $6 \mathrm{~g}$ ).

\section{DISCUSSION}

Our present data provide unprecedentedly comprehensive details of the single-cell mapping of early embryonic VECs in both human and mouse. Specifically, we revealed evolutionarily conserved evident arteriovenous features of early intra-embryonic vasculatures. Importantly, most of the in silico-identified VEC populations here were immunophenotypically and anatomically determined and further transcriptomically validated. The arterial plexus VECs identified here presumably correspond to the pre-artery cells that are defined in the coronary capillary vessels, as both of them are morphologically indistinguishable from their neighboring VECs in the vascular plexus but display apparent arterial characteristics. We also found that they share several novel arterial markers, such as Slc45a4 (Supplementary information, Fig. S8j), Mecom (Fig. 4f), Igfbp3 (Fig. 4c, f), and Ptp4a3 ${ }^{4,10}$ (Fig. 1e; Supplementary information, Fig. S6e).

During mammalian development, the venous-to-arterial fate conversion is known to occur in later stages of organogenesis and be confined to specific organs; e.g., the coronary arteries are initially formed from venous VECs of the sinus venosus. ${ }^{10,11}$ To the best of our knowledge, the arterialization of venous-featured VECs that we revealed here represents the earliest and most extensive fate conversion from venous- to arterial-featured VECs reported to date in mammals. In addition to the fate conversion from venousfeatured VECs revealed here, arterial capillaries might be formed in other potential ways, including de novo generation from angioblasts, sprouting from pre-existing arterial VECs, and arterialized from unspecialized capillary VECs. 3,21,51,52 Except for the initial generation of preliminary vasculature from mesodermal progenitors in early embryos, such as the formation of dorsal aorta, cardinal veins, and vitelline vessels, ${ }^{3,21,51}$ the aortic arch arteries are reported to be derived from the second heart field mesoderm via vasculogenesis at mid-gestation. ${ }^{53-55}$ Whether and to what extent arterial capillaries are directly formed from angioblasts in mammalian embryos is still unclear, which needs further investigations, e.g., with the help of newly developed complicated lineage tracing systems. ${ }^{56,57}$ Notably, although the intersegmental arteries are proposed to be formed by endothelial sprouting from the aortas as evidenced by three-dimensional visualization of the $\mathrm{CD} 31^{+}$vasculature in mouse embryo, ${ }^{19}$ a more rigorous study using genetic lineage-tracing strategy with a Gja5CreER mouse model by tamoxifen induction at E9.5 indicates that Gja5 lineage-labeled VECs are abundantly observed in aortas but seldom detected in the intersegmental arteries at later stages. ${ }^{58}$ Together with our findings, these data highlight the notion that capillary arterialization from venous-featured VECs rather than arterial sprouting from major artery VECs predominantly contributes to the formation of intersegmental arterial VECs. Therefore, our finding reshapes the conventional model regarding the arteriogenesis behaviors of early intra-embryonic vasculatures. The rapid acquisition of arterial characteristics directly in venousfeatured VECs in the vascular plexus of the embryo proper occurs concurrently with the dramatic cellular expansion and precedes the development of major organs in the embryo, ${ }^{17}$ which suggests an efficient way to cooperate with organogenesis. Although embryonic arterial plexus VECs are starting to show an arterial identity on a molecular level, their future fate may not be fixed at this time point, which will depend on specific microenvironment cues. $^{10,12,41,43}$

These findings together will shed new light on the understanding of adult arteriogenesis, especially with regard to the poorly understood process of capillary arterialization, which will have obvious diagnostic and therapeutic impacts for many important diseases and conditions. ${ }^{6}$ The present data also provide an invaluable transcriptomic resource for deeply investigating the mechanisms underlying arteriovenous specification of VECs, which will without doubt pave the way for studies in the vascular regeneration field.

\section{MATERIALS AND METHODS}

\section{Mice}

Mice were handled at the Laboratory Animal Center of Academy of Military Medical Sciences in accordance with institutional guidelines. Mouse manipulations were approved by the Animal Care and Use Committee of the Institute.

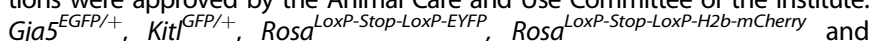
Tie2 ${ }^{\text {Dre/+ }}$ mice were described previously.50,59-62 The Dll4tdTomato/4, Unc5 $b^{\text {tdTomato/+ }}$ and $\mathrm{Nr} 2 \mathrm{f2}^{\text {CrexER/+ }}$ mouse lines were generated with the CRISPR/Cas9 technique by Beijing Biocytogen and Shanghai Model Organisms Center, respectively. All mice were maintained on C57BL/6 background. Embryos were staged by somite pair (sp) counting: E8.0, 1-7 sp; E8.5, 8-12 sp; E9.0, 13-20 sp; E9.5, 21-30 sp; E10.0, 31-35 sp; E10.5, 36-40 sp; and E11.0, 41-45 sp. For well-based scRNA-seq, each embryo was isolated and further dissected into several parts including yolk sac, head, body, and limb buds, excluding heart, visceral bud, and vitelline and umbilical vessels outside embryo proper. For droplet-based scRNA-seq (10x Genomics), embryo proper was collected as a whole excluding heart, visceral bud, and vitelline and umbilical vessels outside embryo proper. In some experiments, caudal half of E10.0 embryo was dissected under heart with limbs removed. The AGM region was dissected as reported. ${ }^{63}$ The dorsal part of caudal half after the AGM region removed was collected as trunk. To specifically capture aortic luminal VECs, we performed microinjection of fluorescent dye Oregon Green into the dorsal aortas of E10.0-E11.0 embryos as reported. ${ }^{63}$ The fluorescent dye Oregon Green 488 was purchased from Invitrogen. Staining was performed as previously described $^{63}$ except that the concentration of staining solution was $5 \mu \mathrm{mol} / \mathrm{L}$ 
and the time of staining was 3 min before washed. Primary embryonic single-cell suspension was acquired by type I collagenase digestion. For lineage tracing, tamoxifen (sigma, T5648) was dissolved in corn oil and administered to pregnant mice by oral gavage at the indicated time. Pregnant mice received $0.1 \mathrm{mg}$ tamoxifen per gram body weight.

\section{Human embryonic sample collection and ethics statement} Human embryonic samples were acquired immediately after elective medical termination of pregnancy in Affiliated Hospital of Academy of Military Medical Sciences (the Fifth Medical Center of the PLA General Hospital). Informed consent in writing was signed before sample collection and all experiments were performed in accordance with protocols approved by the Ethics Committee of the Affiliated Hospital of Academy of Military Medical Sciences (ky-2017- 3-5). Carnegie stages (CS) were used to determine the stages of embryos according to the sp number: CS10, 8-12 sp; CS11, 13-20 sp; CS12, 21-29 sp; CS13, 30-34 sp. For flow cytometric analysis and sorting, head and limb were dissected in the same way as mouse, the rest part of embryos were collected as samples named body. Primary embryonic single-cell suspension was acquired by type I collagenase digestion.

\section{Flow cytometry}

Cells were sorted and analyzed by flow cytometers FACS Aria 2 (BD Biosciences), and the data were analyzed using FlowJo software (Tree Star). For well-based scRNA-seq, the sampling cells were purified by FACS as $\mathrm{CD} 45^{-} \mathrm{CD} 31^{+} \mathrm{CD} 144^{+}$, which contained predominantly VECs and a subset of hematopoietic cells with apparent CD41 expression. Meanwhile, CD45 ${ }^{-} \mathrm{CD} 31^{-} \mathrm{CD} 144^{-}$non-ECs in the body were used as negative controls. For droplet-based scRNA-seq (10x Genomics), only immunophenotypic VEC population $\left(\mathrm{CD} 45^{-} \mathrm{CD} 31^{+} \mathrm{CD} 144^{+}\right)$were collected. Cells from mouse were stained by the following antibodies: CD31 (BD or BioLegend, MEC13.3), CD41 (BD or eBioscience, MWReg30), CD43 (BD, S7), CD44 (eBioscience or BioLegend, IM7), CD45 (eBioscience, 30-F11), CD144 (eBioscience, eBioBV13), DII4 (BioLegend, HMD4-1), CD140b (BioLegend, APB5) and Ki67 (BD). For cell cycle analysis, cells were fixed using Fixation and Permeabilization Solution (BD) after surface marker staining, and then Ki67 and Hoechst (Sigma) staining were performed. Cells from human embryos were stained by the following antibodies: CD31 (BD, WM59), CD34 (BD, 563) and CD45 (BD, 2D1). 7-amino-actinomycin D (7-AAD; eBioscience) was used to exclude dead cells.

\section{Immunofluorescence}

Embryos were isolated, fixed with 4\% paraformaldehyde for $30 \mathrm{~min}$ to $2 \mathrm{~h}$ at $4{ }^{\circ} \mathrm{C}$, embedded in paraffin, and sectioned at 5-6 $\mu \mathrm{m}$ with Leica RM2235. Sections were deparaffinized with ethanol of gradient concentration, then blocked in blocking solution (Zhongshan golden bridge) for $30 \mathrm{~min}$ at room temperature (RT), followed by incubation with primary antibodies overnight at $4{ }^{\circ} \mathrm{C}$. After 3 washes for 3 min each in PBS, sections were incubated with corresponding secondary antibodies (Zhongshan golden bridge) for $30 \mathrm{~min}$ at RT. After 3 washes in PBS, sections were stained with TSA Plus Fluorescein Kit (Histova, NEON 5-color IHC Kit for FFPE, 1:100, 20-60 s). The antibodies were thoroughly eluted by heating the slides in citrate buffer ( $\mathrm{pH} \mathrm{6.0)}$ for $20 \mathrm{~min}$ at $95^{\circ} \mathrm{C}$ using microwave. After that, the next primary antibody was detected following the steps above. Each antigen was labeled by distinct fluorophores. After all the antibodies were detected sequentially, the sections were finally stained with DAPI. Images were collected by confocal microscope (Nikon Ti-E A1/ZEISS LSM 880). The primary antibodies were as follows: aSMA (Cell Signaling, 1:1000), CD36 (Abcam, 1:100), CD44 (BD Biosciences, 1:40), Estrogen Receptor a (Abcam, 1:200), Endomucin (eBioscience, 1:100), GFP (Cell Signaling, 1:200), Ltbp4 (R\&D, 1:400), Nrp2 (Cell Signaling, 1:150), Pdgfr $\beta$ (Cell Signaling, 1:100), CD31 (Cell Signaling, 1:100 or Abcam, 1:1000), ERG (Abcam, 1:100), Runx1 (Abcam, 1:200), BrdU (Abcam, 1:300), and RFP (Rockland, 1:1000).

\section{RNAscope mRNA in situ hybridization assay combined with immunofluorescence}

mRNA expression was determined using double- $Z$ design RNA probes specifically designed to hybridize with Cyp26a1/Ptp4a3 molecules (Advanced Cell Diagnostics, Hayward, CA). RNAscope assay was performed carefully step by step according to the RNAscope ${ }^{\circledR}$ Multiple Fluorescent Reagent Kit v2 Assay (323100-USM; Advanced Cell Diagnostics, Newark, CA). Briefly, tissues were pre-treated with heat and protease prior to hybridization of the target probe. Preamplifier, amplifier and an alkaline phosphatase-labelled oligos were sequentially hybridized followed by the application of TSA Plus Fluorescein Kit (Histova, NEON 4-color IHC Kit for $\mathrm{FFPE}, 1: 300,40^{\circ} \mathrm{C}$ for $20 \mathrm{~min}$ ) to produce punctate dots. After the hybridization, the tissues were blocked with $10 \%$ normal serum in TBS$1 \%$ BSA for $30 \mathrm{~min}$ at RT, followed by the incubation with ERG primary antibody (Abcam) overnight at $4{ }^{\circ} \mathrm{C}$. After 3 washes for 5 min each in TBST, sections were incubated with corresponding HRP-conjugated secondary antibody (Zhongshan golden bridge) for $30 \mathrm{~min}$ at RT. After 3 washes in TBST, sections were stained with TSA Plus Fluorescein Kit (Histova, NEON 4-color IHC Kit for FFPE, 1:100, 1-2 min) and finally stained with DAPI. Images were captured under a confocal fluorescence microscope (ZEISS LSM 880).

\section{BrdU cell proliferation assay}

Mice at E10.0 of gestation were weighed and injected with $0.1 \mu \mathrm{g} / \mathrm{g} \mathrm{BrdU}$. Half an hour after injection, the mice were euthanized via cervical dislocation. Embryos were isolated immediately, fixed with $4 \%$ paraformaldehyde for $2 \mathrm{~h}$ at $4{ }^{\circ} \mathrm{C}$ and embedded in paraffin. BrdU was detected by treating tissue sections with $2 \mathrm{~N} \mathrm{HCl}$ for $20 \mathrm{~min}$ at $37^{\circ} \mathrm{C}$ and incubating BrdU primary antibody (Abcam, 1:100) overnight at $4{ }^{\circ} \mathrm{C}$. After 3 washes for 2 min each in PBS, a corresponding HRP-conjugated secondary antibody (Zhongshan golden bridge) was applied for $30 \mathrm{~min}$ at RT. After 3 washes for 2 min each in PBS, sections were stained with TSA Plus Fluorescein Kit (Histova, NEON 5-color IHC Kit for FFPE, 1:100, 20-60 s). After all antigens were labeled, images were captured on an Akoya Vectra Polaris (PerkinElmer). The numbers of $\mathrm{BrdU}^{+}$endothelial cells in each EP cluster were measured using ImageJ software from merged images.

\section{Retina whole-mount immunostaining}

Mouse retina whole mount immunostaining was prepared as described in previously published standard protocol ${ }^{64}$ with minor modifications. Briefly, after anesthesia, the ocular globes were removed immediately and fixed in the ice-cold $4 \%$ paraformaldehyde for $2 \mathrm{~h}$. Eyes were washed with PBS for at least $5 \mathrm{~min}$, and then the retinas were dissected and cut into four radial incisions under a stereomicroscope (Leica MC170 HD). Dissected retinas were incubated and permeabilized overnight at $4{ }^{\circ} \mathrm{C}$ with blocking buffer (1\% BSA, 0.5\% Triton X-100). The retinas were washed and then incubated with incubation buffer ( $2 \%$ goat serum, $1 \%$ bovine serum albumin (BSA), and $0.5 \%$ Triton $\mathrm{X}-100$ in PBS) for $1 \mathrm{~h}$ at RT, and stained with primary antibody in incubation buffer diluted 1:1 with PBS at $4{ }^{\circ} \mathrm{C}$ overnight Retinas were then washed with PBS and incubated for $2 \mathrm{~h}$ at RT with suitable species-specific IgG (Zhongshan golden bridge) in incubation buffer $(0.5 \%$ bovine serum albumin (BSA), and $0.25 \%$ Triton $X-100$ in PBS). After 3 washes in PBS, retinas were stained with TSA Plus Fluorescein Kit (Histova, NEON 4-color IHC Kit for FFPE, 1:100, 20-60 s). Images were captured under a confocal fluorescence microscope (Nikon Ti-E A1).

\section{Embryo whole-mount immunostaining}

E10.0 embryos were fixed in $2 \%$ paraformaldehyde for $0.5 \mathrm{~h}$ on ice. Immunostaining was performed as described below. Samples were bleached in $5 \% \mathrm{H}_{2} \mathrm{O}_{2}$ for $1 \mathrm{~h}$ on ice to block endogenous peroxidase. After that, samples were incubated for $1 \mathrm{~h}$ in blocking solution $(0.2 \%$ bovine serum albumin, $1 \%$ milk, $0.4 \%(\mathrm{v} / \mathrm{v})$ Triton $\mathrm{X}-100$ in PBS), then incubated overnight at $4{ }^{\circ} \mathrm{C}$ with the designated primary antibodies in blocking solution. After 3 washes for $1 \mathrm{~h}$ each in PBS-MT (1\% milk, 0.4\% (v/v) Triton $\mathrm{X}-100$ in PBS), samples were incubated with suitable species-specific lgG (Zhongshan golden bridge) in PBS-MT overnight at $4{ }^{\circ} \mathrm{C}$. After 3 washes for 20 min each in PBS-T $(0.4 \%(\mathrm{v} / \mathrm{v})$ Triton $\mathrm{X}-100$ in PBS), samples were stained with TSA Plus Fluorescein Kit (Histova, NEON 4-color IHC Kit for Wholemount/Cytometry, 1:500, $20 \mathrm{~min}$ ). Finally, the samples were dehydrated in $100 \%$ methanol, soaked in graded concentrations of BABB (phenylcarbinol and benzyl benzoate, 1:2)/methanol (50\%, 100\%; 1 min each). Images were collected by a confocal microscope (Nikon Ti-E A1/ ZEISS LSM 880).

\section{scRNA-seq library construction}

STRT-seq. For well-based scRNA-seq (modified STRT-seq), single cells in good condition were picked into lysis buffer by mouth pipetting. The scRNA-seq preparation procedure was based on STRT with some modifications. ${ }^{23,65,66}$ cDNAs were synthesized using sample-specific $25-n t$ oligo dT primer containing 8-nt barcode (TCAGACGTGTGCTCTTCCGATCTXXXXXXXX-NNNNNNNN-T25, X representing sample-specific barcode whereas $\mathrm{N}$ standing for unique molecular identifiers (UMIs)) (Supplementary information, Table S8) and template switch oligo (TSO) primer for 
template switching. ${ }^{67-69}$ After reverse transcription and second-strand cDNA synthesis, the CDNAs were amplified by 17 cycles of PCR using ISPCR primer and $3^{\prime}$ Anchor primer (Supplementary information, Table S8). Up to 56 samples were pooled and purified using Agencourt AMPure XP beads (Beckman). Four cycles of PCR were performed to introduce index sequence (Supplementary information, Table S8). After this step, $400 \mathrm{ng}$ cDNAs were fragmented to around 300 bp by covaris S2. The CDNA was incubated with Dynabeads MyOne ${ }^{\mathrm{TM}}$ Streptavidin C1 beads (Thermo Fisher) for $1 \mathrm{~h}$ at RT. Libraries were generated using KAPA Hyper Prep Kit (Kapa Biosystems). After adaptor ligation, the libraries were amplified by 7 cycles of PCR using QP2 primer and short universal primer (Supplementary information, Table S8). The libraries were sequenced on Illumina HiSeq 4000 platform in 150 bp paired-end manner (sequenced by Novogene and Berry Genomics).

10x Genomics. For droplet-based scRNA-seq (10x Genomics), embryos from same stages with hearts removed were pooled together to acquire single-cell suspension. Cells from each stage were sorted and pooled together. Libraries were produced with a Chromium system (10x Genomics, PN120263) following the manufacture's instruction and sequenced on Illumina Hiseq X Ten platform in $150 \mathrm{bp}$ paired-end manner (sequenced by Novogene and Berry Genomics).

\section{Overview of generated scRNA-seq datasets}

We generated multiple VEC-related SCRNA-seq datasets using different technologies (modified STRT-seq and 10X), different species (mouse and human), and different mouse models. Totally, 18,970 single cells were sequenced. Specifically, by using modified STRT-seq method, we generated (i) VEC dataset (Fig. 1b, c; Supplementary information, Table S1, $n=2213$ ) and mural cell dataset (Fig. 3d; Supplementary information, Table S6, $n=432$ ) of mouse wild-type embryos; (ii) 4 validation datasets of 2 mouse reporter models of Dll4-tdTom (Fig. 2d; Supplementary information, Table S5, $n=288$ ) and Unc5b-tdTom (Fig. 2d; Supplementary information, Table S5, $n=144$ ), and 2 mouse lineage-tracing models of Tie2Nr2f2-Cre;Rosa-EYFP;Unc5b-tdTom (Fig. 6d; Supplementary information, Table S5, $n=192$ ) and Nr2f2-CrexER;Rosa-H2b-mCherry;Gja5-EGFP (Fig. 6f; Supplementary information, Table S5, $n=112$ ); and (iii) human embryo VEC dataset (Fig. 4a; Supplementary information, Table S7, $n=996$ ). In addition, we also generated (iv) VEC 10x dataset (Supplementary information, Fig. S3 and Table S4, $n=10,465$ ) of mouse wild-type embryos using $10 \times$ Genomics method.

\section{Processing of scRNA-seq raw data}

STRT-seq. For modified STRT-seq data, we used UMI-based scRNA-seq method to accurately measure the gene expression profiles within individual cells. Raw reads of each cell were first split by specific barcode attached in Read 2. The corresponding Read 1 was trimmed to remove the TSO sequence and polyA tail sequence after UMI information was aligned to it. Subsequently, we conducted quality control to discard reads with adapter contaminants or low-quality bases $(N>10 \%)$. Next, the $\mathrm{mm} 10$ (for mouse data) or hg19 (for human data) transcriptome (UCSC) was used to align the clean reads through TopHat (version 2.0.12). ${ }^{70}$ Uniquely mapped reads were obtained by HTSeq package ${ }^{71}$ and grouped by the cell-specific barcodes. Duplicated transcripts of each gene were removed based on the UMI information. For each individual cell, the copy number of transcripts of a given gene was the number of the distinct UMls of that gene. Finally, expression levels were normalized by $\log (T P M / 10+1)$, where TPM (transcripts-per-million) was calculated as (the number of UMls of each gene/all UMls of a given cell) $\times 1,000,000$. Since the UMI number of most of our samples was less than the order of 1,000,000 transcripts, the TPM values were divided by 10 to avoid counting each transcript for several times.

$10 \times$ Genomics. Sequencing data from 10x Genomics were processed with the Cell Ranger software (version 2.1.0) for each sample. Data were mapped to the $\mathrm{mm} 10$ (for mouse data) or hg19 (for human data) reference data (version 1.2.0) downloaded from 10x Genomics official website. The UMI data matrixes of quantitative gene expression for each sample were obtained. Expression levels were normalized by $\log ($ TPM/100 +1$)$, since the UMI number of most of our samples sequenced by using $10 \times$ Genomics method was on the order of 10,000 transcripts.

\section{Quality control of scRNA-seq data}

STRT-seq. We applied slightly different but strict criteria to different datasets, taking into account the different sequencing depths. For mouse
VEC and MC datasets, respectively, 2066 (93.4\%) and 400 (92.6\%) single cells with more than 2000 genes and 100,000 transcripts detected were retained. For 3 mouse validation datasets of Dll4-tdTom, Unc5b-tdTom, and Tie2Nr2f2-Cre;Rosa-EYFP;Unc5b-tdTom models, only cells with more than 2000 genes and 50,000 transcripts detected were retained. Then 274 (95.1\%), $131(91.0 \%)$ and $174(90.6 \%)$ single cells, respectively for each dataset, passed the filter standards. For mouse validation dataset of the Nr2f2-CrexER;Rosa-H2b-mCherry;Gja5-EGFP model, 85 (75.9\%) single cells with more than 1000 genes and 10,000 transcripts detected were retained. For human VEC dataset, 835 (86.4\%) single cells with more than 2000 genes and 10,000 transcripts detected were retained.

10x Genomics. Filtered cells by Cell Ranger from VEC 10x dataset of mouse wild-type embryos were retained. Data from two biological replicates were concatenated into a single dataset including 12,146 cells. We removed 1681 cells from further analyses as they clustered separately from the main group and did not express any distinct markers. Based on their expression profiles, these cells were probably low-quality cells.

\section{Seurat analysis procedure for scRNA-seq datasets}

Seurat (version 3.1.5) ${ }^{72,73}$ was employed for advanced analysis of STRT-seq datasets. For different STRT-seq datasets, we followed the similar Seurat analysis procedure. Briefly, we first used 'SCTransform' function to perform the workflow of data normalization, identification of highly variable genes (HVGs) and data scaling, and then used 'RunPCA' function to perform PCA dimensionality reduction. Selected top PCs were retained using the elbow method for computing the UMAP dimensional reduction ${ }^{74}$ and constructing a Shared Nearest Neighbor (SNN) Graph. We used a SNN modularity optimization-based clustering algorithm, implemented in 'FindClusters' function, to identify clusters of cells. Parameter 'resolution' was used to control the number of clusters of cells.

\section{Analysis of VEC dataset from mouse wild-type embryos}

STRT-seq. Following the Seurat analysis procedure described above, we initially achieved three major groups, namely VEC, Hematopoietic cell and Non-EC (Supplementary information, Fig. S2a and Table S1). Then, some cells that were not in the scope of our interest were excluded for further analysis: (i) negative control cells with a non-EC immunophenotype, (ii) cells belonging to groups of Hematopoietic cell and Non-EC, (iii) cells with Ptprc expression level of $\log (\mathrm{TPM} / 10+1)$ greater than 1, and (iv) allantoic VECs, which were identified by unsupervised clustering of all cells from E8.0-E8.5 and expression of marker genes (e.g., Hoxa10 and Hoxa11). We then redid the Seurat analysis procedure to cluster the cells into yolk sac VEC group and embryo proper VEC group. Next, we further clustered the embryo proper VEC group into eight EP clusters (EPO-EP7) and the yolk sac VEC group into three YS clusters (YS1-YS3), using the Seurat analysis procedure. Notably, given the relatively limited heterogeneity and the nature of molecular continuum of the cells in EP6 and EP7, we employed a method reported previously ${ }^{75}$ with some modifications to optimize the clustering results between EP6 and EP7. This method combined unsupervised clustering to reveal heterogeneity in cell subtypes and supervised classification to fine-tune clusters. In brief: (i) we first identified differentially expressed genes (DEGs) between EP6 and EP7 using 'FindAllMarkers' function; (ii) we then performed hierarchical clustering through Pearson correlation distance metrics and got two clusters at the first split; (iii) to select feature genes dividing the two clusters, we performed a 10-fold random forest feature selection; (iv) to adjust initial classifications, we selected samples with internal vote probabilities $>0.6$ for each class as the training set to achieve an optimal classifier, which was used to predict the rest of the samples; (v) we performed 100 runs of 10-fold random forest cross validation (CV) and discarded samples with internal vote probabilities $<0.55$. We used internal vote probabilities $>0.55$ (default $=0.5$ ) as the cutoff to reduce the ambiguity of sample voting. For example, if the probability of assigning a sample to cluster A is $51 \%$ and to cluster B is $49 \%$, we assume the sample is ambiguous to be classified to cluster $A$ and need to be eliminated during classification. We used default parameters for number of trees. In this way, fine-tuned clusters of EP6 and EP7 were identified.

10x Genomics. Downstream bioinformatics analyses were done with Scanpy (version 1.4.3). ${ }^{76}$ The data were normalized using standard totalcount normalization and then taken $\log 1 \mathrm{p}$ transformation as expression level. Genes with normalized dispersion $>0.5$ and average expression $>0.5$ but $<5$ were selected as HVGs. The expression data was scaled with unit variance and zero mean. The scaled data of HVGs were used for PCA. Top 
25 PCs were retained using the elbow method for computing the neighborhood graph and embedding the neighborhood graph using UMAP. ${ }^{74}$ Finally, Louvain algorithm ${ }^{77}$ was employed to cluster cells into subgroups. DEGs were identified using 'logreg' method. ${ }^{78}$

\section{Identification of DEGs}

STRT-seq. We used the FindAllMarkers function and FindMarkers function in Seurat to identify cluster feature genes between multiple clusters and DEGs between two given clusters, respectively. The $\log (\mathrm{TPM} / 10+1)$ transformed expression values and 'wilcox' method were used. Only genes that are detected in a minimum fraction of 0.25 cells in either of two populations were included for test. Genes with fold-change $\geq 2$ and adjusted $P$ value $\leq 0.05$ were retained as DEGs.

10x Genomics. DEGs were identified using 'logreg' method ${ }^{78}$ implemented in Scanpy.

\section{GO enrichment analysis}

Strict thresholds were used for identification of specific marker genes between clusters as described above in DEG analysis, which resulted in fewer genes (less than 100 DEGs in most cases) being retained. To achieve adequate number of DEGs for enrichment analysis, we tuned down the threshold of fold-change to 1.5. Then the top 200 DEGs (if any) were used for subsequent analysis. Network enrichment analysis was performed using Metascape ${ }^{79}$ (http://metascape.org). GO biological process enrichment analysis was performed using clusterProfiler. ${ }^{80}$

\section{GSEA/KEGG analysis}

Gene Set Enrichment Analysis (GSEA) is a widely used computational analysis method that determines whether a predefined gene set shows statistically significant, concordant differences between two biological states. GSEA coupled with the gene set data of Kyoto Encyclopedia of Genes and Genomes (KEGG) was applied to explore signaling pathways changes by pairwise comparisons of two selected clusters. The same procedure was used for each comparison. Briefly, KEGG pathway gene sets were downloaded from Molecular Signatures Database (MSigDB version 6.1, http://www.broadinstitute.org/gsea/index.jsp) and getLDS function in biomaRt $\mathrm{R}$ package ${ }^{81}$ was employed to convert human genes to mouse homologous genes. Then we used the javaGSEA 4.1.0 with permutation 10,000 and Signal2Noise as metrics for ranking genes. We chose FDR $<0.1$ to select the significantly altered pathways. Normalized enrichment scores (NES) of all significant pathways were used for illustration in heatmap, and NESs of insignificant pathways were set to zero for the sake of simplification. Hierarchical clustering of pathways and comparisons were performed on indicator matrix of pathway change direction, using Euclidean as distance metric and ward.D as agglomeration method. Indicator of pathway change direction was defined as 0 if pathway was insignificantly changed, otherwise, 1 if NES was positive and -1 if negative.

\section{Arterial and venous feature score}

We selected a set of arteriovenous marker genes previously known or inferred from the artery development pattern genes, including 13 arterial genes $(B m x$, Cxcr4, Dll4, Efnb2, Epas1, Gja4, Gja5, Hey1, Igfbp3, Mecom, Nrp1, Unc5b and Vegfc) and 3 venous genes (Aplnr, Nr2f2 and Nrp2), ${ }^{6,10,21,37}$ to perform the arteriovenous feature score. First, we scaled the $\log _{2}(T P M / 10+1)$ expression values of each of the 20 marker genes to $0-10$ scale among all the sample cells after quality control. Second, for each cell, we averaged the scaled values of arterial genes and venous genes, respectively. Third, the averaged values were rescaled to $0-10$ scale across all the sample cells to finally achieve the arterial and venous score, respectively. For each population, the arterial and venous scores of all of the cells within the population were averaged, respectively, and the $50 \%$ confidence ellipse was also calculated to show the main distribution range. We chose score 5 as the threshold to infer the arterial or venous identity of VECs, as the distribution of individual cells was in line with the notion showing essentially no arterial/ venous double-positive cells. In addition, homologous genes of the set of arteriovenous marker genes were used for this analysis of human VEC dataset.

\section{Fitting principal curve in PCA space}

A principal curve is a smooth curve passing through the middle of a multidimensional dataset. Combined with PCA, principal curve may model mainly the gradual changes associated with cell differentiation or lineage relationships from single-cell gene expression data. ${ }^{82}$ In brief, (i) we used standard PCA to get default PC scores; (ii) we replaced default principal component scores with a sum of the top 60 gene scores because it has been proven to be less correlated with technical artefact and better correlated with cluster-specific genes; ${ }^{10}$ (iii) we applied the princurve $\mathrm{R}$ package $^{82}$ to the top two PCs, the most informative dimensions underlying the transcriptome, for fitting a principal curve and inferring the pseudoorder of cells. We applied this workflow to infer the cell pseudo-order for all cells belonging to selected cell populations, such as clusters of EP1 and EP2, clusters of EP2 and EP3, clusters of EP6 and EP7 (Fig. 3a; Supplementary information, Fig. S6a).

\section{Identification of pattern genes of arterial vasculature}

We presumed that VECs included in EP1 and EP2 was a continuum of arterial-venous identity, VECS in EP2 and EP3 were involved in the development of major arteries and VECs in EP6 and EP7 was a continuum of later vascular plexus. Up-regulated genes in EP2 compared to EP1 were assumed as arterial segregation genes, and up-regulated genes in EP3 compared to EP2 were assumed as arterial strengthening genes. These DEGs were identified using 'wilcox' method with fold change $>1.5$ as threshold (Supplementary information, Table S3). For visualization of changes in gene expression across these spatially or temporally continuous VEC populations, we inferred the pseudo-order of cells involved in each continuum, using the method described in 'Fitting principal curve in PCA space'. Such analysis was performed on VEC populations of EP1 \& EP2, EP2 \&EP3, and EP6 \& EP7, respectively. All cells within the given clusters were included for analysis, regardless of sampling locations and stages. In the visualizations, cells are ordered by pseudo-order, and pattern genes are ordered firstly by pattern categories and then by $P$ values of comparison between EP6 and EP7 using Wilcoxon rank sum test.

\section{Cell cycle analysis}

We used CellCycleScoring function in Seurat to calculate the cell cycle phase scores and achieve cell cycle phase assignments for single cells. Cell cycle-related genes were used, including a previously defined core set of $43 \mathrm{G} 1 / \mathrm{S}$ and 54 G2/M genes ${ }^{69,83}$ (Supplementary information, Table S8).

\section{Putative interactions between endothelial cells and mural cells}

We identified putative interactions between VECs with an arterial feature and their corresponding mural cells using the gene sets of potential receptor-ligand interactions collected from Ramilowski et al. ${ }^{84}$ Based on the spatiotemporal relationships, we defined three pairs of datasets, namely, E9.5 DA-derived mural cells vs cluster EP3 VECs, E10.5 DA-derived mural cells vs cluster EP5 VECs, and E9.5 limb bud-derived mural cells vs cluster EP7 VECs. We used log(TPM/10 + 1) transformed expression values to measure the potential interactions. If the expression value of a certain receptor in more than $50 \%$ cells of one cell type exceeded 1.34 , and the expression value of the matched ligand in more than $50 \%$ cells of the other cell type also exceeded 1.34, we regarded this ligand-receptor pair as a potential heterologous cellular interaction between these two cell types (Supplementary information, Table S6). The expression threshold of 1.34 is determined by the upper quartile of expression values of all genes in the analyzed data.

\section{Constructing single cell trajectories}

To infer the developing trajectory of individual cells or cell populations, we adopted 3 methods, namely Mpath, ${ }^{48}$ monocle $3^{17}$ and scVelo (version $0.2 .1)^{85}$ to ensure the accuracy of trajectory analyses. For Mpath analysis, the HVGs identified using routine analysis procedure described above, were used. The cluster labels produced by our clustering procedures were used as landmark cluster assignment of individual cells, and other parameters used the default values (see https://github.com/ JinmiaoChenLab/Mpath). For scVelo analysis, we used the steady-state model, as being used in velocyto ${ }^{49}$ to estimate RNA velocities of single cells by distinguishing unspliced and spliced mRNAs. We used velocyto command line interface to generate spliced/unspliced expression matrices and scVelo package to estimate velocity following the tutorial (https:// scvelo.readthedocs.io/VelocityBasics.html). Then, we visualized RNA velocity vector field of the most fine-grained resolution on the UMAP embedding space generated by Seurat analysis. For Monocle 3 analysis, we also used the UMAP embeddings by Seurat to learn the trajectory 
graph and order the cells in pseudotime following the tutorial (https://coletrapnell-lab.github.io/monocle3/docs/trajectories/). We used order_cells function to achieve the branch assignments and pseudotime values of cells by manually specifying the root nodes and end nodes in the principal trajectory graph. The root node was determined based on the sampling stage and results from other trajectory analyses.

\section{Integration analysis using fast-Mutual Nearest Neighbors (fastMNN)}

We employed the fastMNN correction algorithm ${ }^{86}$ to mitigate batch effects (e.g., from different mouse models or different species) and perform data integration analysis for validation datasets or human datasets. Briefly, we used RunFastMNN function of SeuratWrappers $\mathrm{R}$ package to integrate datasets derived from different species, genotypes and/or library construction strategies. Then selected top dimensions in MNN space were retained for computing the UMAP dimensional reductions. Specifically, for integration of mouse VEC dataset and each validation dataset (Figs. 2d, e and $6 \mathrm{~d}, \mathrm{f}), \mathrm{HVGs}$ identified in mouse VEC dataset were used as inputs.

\section{Analysis of VEC dataset from human embryos}

We performed the same Seurat analysis procedure as described above, except that the batch effects and cell cycle phase scores of S.Score and G2M. Score were regressed out using the SCTransform function. Because both batch effects and cell cycle effects dominantly affect the dimension reduction results of human VEC dataset, which is different from the scene of mouse datasets. Then we achieved seven clusters of VEC1, VEC2, GATA4 ${ }^{+}-$ $\mathrm{HAND}_{2}{ }^{+}, \mathrm{GATA}_{4}^{+} \mathrm{OIT}^{+}$, Hemogenic endothelial cell, Hematopoietic cell and Non-EC (Supplementary information, Fig. S8d and Table S7). Clusters of VEC1 and VEC2 were retained as VEC populations for further analysis.

Given the limited number of early VECs in human dataset, due to scarcity of human earlier embryos, we took mouse VEC dataset as reference for integration analysis to better understand conservation of VEC development in mammals. We integrated human VECs with mouse VECs in embryo proper VEC group by using the fastMNN integration analysis procedure. Specifically, mouse genes were converted into human homologous genes for combining the datasets from different species by using getLDS function in biomaRt $R$ package. ${ }^{81}$ Considering that the cell cycle effects dominantly affect the dimension reduction results, we first select HVGs using SelectlntegrationFeatures function, and then excluded the genes highly correlated with cell cycle scores (absolute correlation coefficient $>0.3$ ). HVGs after excluding putative cell cycle genes were used for the integration procedure, which largely moderated the impact of cell cycle effects on dimension analysis. In the integrated MNN space, we classified human VECs into the seven VEC clusters (EP0-EP3 and EP5-EP7) of mouse VEC reference dataset, by using k-Nearest Neighbour classification method.

We also used the same fastMNN analysis procedure described above to integrate VECs derived from three datasets of human VEC dataset generated by STRT-seq and mouse VEC datasets generated by both $10 \times$ Genomics and STRT-seq.

\section{Conversed genes between two species during vascular development}

We focused on the two types of arterial VEC populations for further analysis. We renamed the human EP3 and EP5 combination as arterial VEC1 (hAEC1), EP7 as $\mathrm{hAEC}$, and EP6 as venous VEC ( $\mathrm{hVeEC),} \mathrm{corresponding} \mathrm{to} \mathrm{the} \mathrm{mouse}$ EP3 and EP5 combination (major artery VECs, MAEC1), EP7 (arterial plexus VECs, mAEC2), and EP6 (vein \& venous plexus VECs, mVeEC), respectively. We determined the conserved genes as shared DEGs in each cluster pair between two species. For mouse dataset, getLDS function in biomaRt $R$ package ${ }^{81}$ was employed to convert mouse genes to human homologous genes. We also made an effort to identify conserved arterial genes, which were defined as those highly expressed in both arterial VEC populations (hAEC1/mAEC1 and hAEC2/mAEC2), when respectively compared with the corresponding venous VEC population $\mathrm{hVeEC} / \mathrm{mVeEC}$. We used Wilcoxon rank sum test to calculate the significance of each gene, and only genes with fold change $>2$ and adjusted $P$ value $<0.05$ were retained as DEGs. Fisher's method was employed to combine two or multiple independent $P$ values into a single combined $P$ value.

\section{Dynamically changed genes along arterial VEC development in both human and mouse}

We applied Monocle 3 in the integrated UMAP space composed of human and mouse VECs, and achieved trajectories of two types of arterial VEC development for human and mouse simultaneously. Then, identification of pattern genes changed along trajectories were performed for human and mouse VECs, respectively. Briefly, pattern genes changed along each development path were identified by using differentialGeneTest function in Monocle $2 .^{87}$ Top 500 significantly changed genes were retained for visualization in heatmaps for each development path. Pattern genes were clustered using kmeans method, and the number of clusters were determined by manually checking the heatmap results from larger to smaller number of clusters. Then pattern diagrams were illustrated, and enriched GO terms of genes for different patterns were calculated.

\section{Transcription factors}

Transcription factors used in this study including 1691 human transcription factors and 1485 mouse transcription factors were download from Animal TFDB (http://bioinfo.life.hust.edu.cn/AnimalTFDB/) ${ }^{88}$ (Supplementary information, Table S8).

\section{Statistical analysis}

All statistical analyses were conducted in $\mathrm{R}$ language. Two-sample Wilcoxon Rank Sum test was employed for comparisons of gene numbers, transcript counts, gene expression levels and feature scores between two given clusters of cells. More detailed information of statistical analysis is described above.

\section{DATA AVAILABILITY}

All of the scRNA-seq data have been deposited in Gene Expression Omnibus under the accession number GSE94877.

\section{CODE AVAILABILITY}

All data were analyzed with standard programs and packages, as detailed above. Code is available on request.

\section{REFERENCES}

1. Adams, R. H. \& Alitalo, K. Molecular regulation of angiogenesis and lymphangiogenesis. Nat. Rev. Mol. Cell Biol. 8, 464-478 (2007).

2. Herbert, S. P. \& Stainier, D. Y. Molecular control of endothelial cell behaviour during blood vessel morphogenesis. Nat. Rev. Mol. Cell Biol. 12, 551-564 (2011).

3. Potente, M. \& Makinen, T. Vascular heterogeneity and specialization in development and disease. Nat. Rev. Mol. Cell Biol. 18, 477-494 (2017).

4. Red-Horse, K. \& Siekmann, A. F. Veins and Arteries Build Hierarchical Branching Patterns Differently: Bottom-Up versus Top-Down. BioEssays 41, e1800198 (2019).

5. Faber, J. E., Chilian, W. M., Deindl, E., van Royen, N. \& Simons, M. A brief etymology of the collateral circulation. Arterioscler Thromb. Vasc. Biol. 34, 1854-1859 (2014).

6. Simons, M. \& Eichmann, A. Molecular controls of arterial morphogenesis. Circ. Res. 116, 1712-1724 (2015).

7. Tian, X. et al. Vessel formation. De novo formation of a distinct coronary vascular population in neonatal heart. Science 345, 90-94 (2014).

8. Fujita, M. et al. Assembly and patterning of the vascular network of the vertebrate hindbrain. Development 138, 1705-1715 (2011).

9. Bussmann, J., Wolfe, S. A. \& Siekmann, A. F. Arterial-venous network formation during brain vascularization involves hemodynamic regulation of chemokine signaling. Development 138, 1717-1726 (2011).

10. Su, T. et al. Single-cell analysis of early progenitor cells that build coronary arteries. Nature 559, 356-362 (2018).

11. Red-Horse, K., Ueno, H., Weissman, I. L. \& Krasnow, M. A. Coronary arteries form by developmental reprogramming of venous cells. Nature 464, 549-553 (2010).

12. $\mathrm{Xu}, \mathrm{C}$. et al. Arteries are formed by vein-derived endothelial tip cells. Nat. Commun. 5, 5758 (2014).

13. Kalucka, J. et al. Single-cell transcriptome atlas of murine endothelial cells. Cell 180, 764-779.e720 (2020).

14. Paik, D. T. et al. Single-cell RNA sequencing unveils unique transcriptomic signatures of organ-specific endothelial cells. Circulation 142, 1848-1862 (2020)

15. Vanlandewijck, M. et al. A molecular atlas of cell types and zonation in the brain vasculature. Nature 554, 475-480 (2018).

16. Pijuan-Sala, B. et al. A single-cell molecular map of mouse gastrulation and early organogenesis. Nature 566, 490-495 (2019).

17. Cao, J. et al. The single-cell transcriptional landscape of mammalian organogenesis. Nature 566, 496-502 (2019).

18. Drake, C. J. \& Fleming, P. A. Vasculogenesis in the day 6.5 to 9.5 mouse embryo. Blood 95, 1671-1679 (2000). 
19. Walls, J. R., Coultas, L., Rossant, J. \& Henkelman, R. M. Three-dimensional analysis of vascular development in the mouse embryo. PLoS ONE 3, e2853 (2008).

20. Dzierzak, E. \& Speck, N. A. Of lineage and legacy: the development of mammalian hematopoietic stem cells. Nat. Immunol. 9, 129-136 (2008).

21. Chong, D. C., Koo, Y., Xu, K., Fu, S. \& Cleaver, O. Stepwise arteriovenous fate acquisition during mammalian vasculogenesis. Dev. Dyn. 240, 2153-2165 (2011).

22. Takase, $\mathrm{H}$. et al. Genome-wide identification of endothelial cell-enriched genes in the mouse embryo. Blood 120, 914-923 (2012).

23. Li, L. et al. Single-cell RNA-seq analysis maps development of human germline cells and gonadal niche interactions. Cell Stem Cell 20, 891-892 (2017).

24. Scialdone, A. et al. Resolving early mesoderm diversification through single-cell expression profiling. Nature 535, 289-293 (2016).

25. McGrath, K. E. et al. Distinct sources of hematopoietic progenitors emerge before HSCs and provide functional blood cells in the mammalian embryo. Cell Rep. 11, 1892-1904 (2015).

26. Ibarra-Soria, X. et al. Defining murine organogenesis at single-cell resolution reveals a role for the leukotriene pathway in regulating blood progenitor formation. Nat. Cell Biol. 20, 127-134 (2018).

27. Rasmussen, T. L. et al. ER71 directs mesodermal fate decisions during embryogenesis. Development 138, 4801 (2011).

28. Lu, X. et al. The netrin receptor UNC5B mediates guidance events controlling morphogenesis of the vascular system. Nature 432, 179-186 (2004).

29. Wheatley, S. C., Isacke, C. M. \& Crossley, P. H. Restricted expression of the hyaluronan receptor, CD44, during postimplantation mouse embryogenesis suggests key roles in tissue formation and patterning. Development 119, 295-306 (1993).

30. Zilberberg, L. et al. Specificity of latent TGF-beta binding protein (LTBP) incorporation into matrix: role of fibrillins and fibronectin. J. Cell Physiol. 227, 3828-3836 (2012).

31. Papke, C. L. \& Yanagisawa, H. Fibulin-4 and fibulin-5 in elastogenesis and beyond: Insights from mouse and human studies. Matrix Biol. 37, 142-149 (2014).

32. Bruzzone, R., Haefliger, J. A., Gimlich, R. L. \& Paul, D. L. Connexin40, a component of gap junctions in vascular endothelium, is restricted in its ability to interact with other connexins. Mol. Biol. Cell 4, 7-20 (1993).

33. Vincent, S. D. et al. The zinc finger transcriptional repressor Blimp1/Prdm1 is dispensable for early axis formation but is required for specification of primordial germ cells in the mouse. Development 132, 1315-1325 (2005).

34. Wagenseil, J. E. \& Mecham, R. P. Vascular extracellular matrix and arterial mechanics. Physiol. Rev. 89, 957-989 (2009).

35. Thambyrajah, R. et al. GFI1 proteins orchestrate the emergence of haematopoietic stem cells through recruitment of LSD1. Nat. Cell Biol. 18, 21-32 (2016).

36. Hou, S. et al. Embryonic endothelial evolution towards first hematopoietic stem cells revealed by single-cell transcriptomic and functional analyses. Cell Res. 30, 376-392 (2020)

37. Corada, M., Morini, M. F. \& Dejana, E. Signaling pathways in the specification of arteries and veins. Arterioscler Thromb. Vasc. Biol. 34, 2372-2377 (2014).

38. Barnes, R. M. et al. Hand2 loss-of-function in Hand1-expressing cells reveals distinct roles in epicardial and coronary vessel development. Circ. Res. 108, 940-949 (2011)

39. Ehling, M., Adams, S., Benedito, R. \& Adams, R. H. Notch controls retinal blood vessel maturation and quiescence. Development 140, 3051-3061 (2013).

40. Chu, M. et al. Angiopoietin receptor Tie2 is required for vein specification and maintenance via regulating COUP-TFII. Elife 5, e21032 (2016).

41. Pitulescu, M. E. et al. Dll4 and Notch signalling couples sprouting angiogenesis and artery formation. Nat. Cell Biol. 19, 915-927 (2017)

42. Fang, J. S. et al. Shear-induced Notch-Cx37-p27 axis arrests endothelial cell cycle to enable arterial specification. Nat. Commun. 8, 2149 (2017).

43. Luo, W. et al. Arterialization requires the timely suppression of cell growth. Nature 589, 437-441 (2021).

44. Armulik, A., Genove, G. \& Betsholtz, C. Pericytes: developmental, physiological, and pathological perspectives, problems, and promises. Dev. Cell 21, 193-215 (2011).

45. Oberlin, E., Tavian, M., Blazsek, I. \& Peault, B. Blood-forming potential of vascular endothelium in the human embryo. Development 129, 4147-4157 (2002).

46. Zeng, Y. et al. Tracing the first hematopoietic stem cell generation in human embryo by single-cell RNA sequencing. Cell Res. 29, 881-894 (2019).

47. MacParland, S. A. et al. Single cell RNA sequencing of human liver reveals distinct intrahepatic macrophage populations. Nat. Commun. 9, 4383 (2018).

48. Chen, J., Schlitzer, A., Chakarov, S., Ginhoux, F. \& Poidinger, M. Mpath maps multibranching single-cell trajectories revealing progenitor cell progression during development. Nat. Commun. 7, 11988 (2016).

49. La Manno, G. et al. RNA velocity of single cells. Nature 560, 494-498 (2018).

50. Pu, W. et al. Genetic targeting of organ-specific blood vessels. Circ. Res. 123, 86-99 (2018).

51. Fish, J. E. \& Wythe, J. D. The molecular regulation of arteriovenous specification and maintenance. Dev. Dyn. 244, 391-409 (2015).
52. He, L. et al. Genetic lineage tracing discloses arteriogenesis as the main mechanism for collateral growth in the mouse heart. Cardiovasc. Res. 109, 419-430 (2016).

53. Li, P., Pashmforoush, M. \& Sucov, H. M. Mesodermal retinoic acid signaling regulates endothelial cell coalescence in caudal pharyngeal arch artery vasculogenesis. Dev. Biol. 361, 116-124 (2012).

54. Wang, X. et al. Endothelium in the pharyngeal arches 3, 4 and 6 is derived from the second heart field. Dev. Biol. 421, 108-117 (2017).

55. Kelly, R. G. Integrating matrix signals during arch artery morphogenesis. Circ. Res. 128, 360-362 (2021).

56. Han, X. et al. A suite of new Dre recombinase drivers markedly expands the ability to perform intersectional genetic targeting. Cell Stem Cell 28, 1160-1176 e1167 (2021).

57. He, L. et al. Enhancing the precision of genetic lineage tracing using dual recombinases. Nat. Med. 23, 1488-1498 (2017)

58. Beyer, S., Kelly, R. G. \& Miquerol, L. Inducible Cx40-Cre expression in the cardiac conduction system and arterial endothelial cells. Genesis 49, 83-91 (2011).

59. Miquerol, L. et al. Architectural and functional asymmetry of the His-Purkinje system of the murine heart. Cardiovasc. Res. 63, 77-86 (2004).

60. Ding, L., Saunders, T. L., Enikolopov, G. \& Morrison, S. J. Endothelial and perivascular cells maintain haematopoietic stem cells. Nature 481, 457-462 (2012).

61. Srinivas, S. et al. Cre reporter strains produced by targeted insertion of EYFP and ECFP into the ROSA26 locus. BMC Dev. Biol. 1, 4 (2001).

62. Yue, Y. et al. Long-term, in toto live imaging of cardiomyocyte behaviour during mouse ventricle chamber formation at single-cell resolution. Nat. Cell Biol. 22, 332-340 (2020).

63. Rybtsov, S. et al. Hierarchical organization and early hematopoietic specification of the developing HSC lineage in the AGM region. J. Exp. Med. 208, 1305-1315 (2011).

64. Pitulescu, M. E., Schmidt, I., Benedito, R. \& Adams, R. H. Inducible gene targeting in the neonatal vasculature and analysis of retinal angiogenesis in mice. Nat. Protoc. 5, 1518-1534 (2010).

65. Picelli, S. et al. Smart-seq2 for sensitive full-length transcriptome profiling in single cells. Nat. Methods 10, 1096-1098 (2013).

66. Picelli, S. et al. Full-length RNA-seq from single cells using Smart-seq2. Nat. Protoc. 9, 171-181 (2014).

67. Hashimshony, T., Wagner, F., Sher, N. \& Yanai, I. CEL-Seq: single-cell RNA-Seq by multiplexed linear amplification. Cell Rep. 2, 666-673 (2012).

68. Islam, S. et al. Quantitative single-cell RNA-seq with unique molecular identifiers. Nat. Methods 11, 163-166 (2014).

69. Macosko, E. Z. et al. Highly parallel genome-wide expression profiling of individual cells using nanoliter droplets. Cell 161, 1202-1214 (2015).

70. Trapnell, C., Pachter, L. \& Salzberg, S. L. TopHat: discovering splice junctions with RNA-Seq. Bioinformatics 25, 1105-1111 (2009).

71. Anders, S., Pyl, P. T. \& Huber, W. HTSeq-a Python framework to work with highthroughput sequencing data. Bioinformatics 31, 166-169 (2015).

72. Butler, A., Hoffman, P., Smibert, P., Papalexi, E. \& Satija, R. Integrating single-cell transcriptomic data across different conditions, technologies, and species. Nat. Biotechnol. 36, 411-420 (2018).

73. Stuart, T. et al. Comprehensive integration of single-cell data. Cell 177 1888-1902.e1821 (2019).

74. McInnes, L., Healy, J. \& Melville, J. Umap: Uniform manifold approximation and projection for dimension reduction. arXiv:1802.03426 (2018).

75. Lake, B. B. et al. Neuronal subtypes and diversity revealed by single-nucleus RNA sequencing of the human brain. Science 352, 1586-1590 (2016).

76. Wolf, F. A., Angerer, P. \& Theis, F. J. SCANPY: large-scale single-cell gene expression data analysis. Genome Biol. 19, 15 (2018).

77. Levine, J. H. et al. Data-driven phenotypic dissection of AML reveals progenitorlike cells that correlate with prognosis. Cell 162, 184-197 (2015).

78. Ntranos, V., Yi, L., Melsted, P. \& Pachter, L. A discriminative learning approach to differential expression analysis for single-cell RNA-seq. Nat. Methods 16, 163-166 (2019).

79. Tripathi, S. et al. Meta- and orthogonal integration of influenza "OMICs" data defines a role for UBR4 in virus budding. Cell Host Microbe 18, 723-735 (2015).

80. Yu, G., Wang, L. G., Han, Y. \& He, Q. Y. clusterProfiler: an R package for comparing biological themes among gene clusters. OMICS 16, 284-287 (2012).

81. Durinck, S., Spellman, P. T., Birney, E. \& Huber, W. Mapping identifiers for the integration of genomic datasets with the R/Bioconductor package biomaRt. Nat. Protoc. 4, 1184-1191 (2009).

82. Hastie, T. \& Stuetzle, W. Principal curves. J. Am. Statist. Assoc. 84, 502-516 (1989).

83. Tirosh, l. et al. Dissecting the multicellular ecosystem of metastatic melanoma by single-cell RNA-seq. Science 352, 189-196 (2016).

84. Ramilowski, J. A. et al. A draft network of ligand-receptor-mediated multicellular signalling in human. Nat. Commun. 6, 7866 (2015). 
348

85. Bergen, V., Lange, M., Peidli, S., Wolf, F. A. \& Theis, F. J. Generalizing RNA velocity to transient cell states through dynamical modeling. Nat. Biotechnol. 38, 1408-1414 (2020).

86. Haghverdi, L., Lun, A. T. L., Morgan, M. D. \& Marioni, J. C. Batch effects in singlecell RNA-sequencing data are corrected by matching mutual nearest neighbors. Nat. Biotechnol. 36, 421-427 (2018).

87. Qiu, X. et al. Reversed graph embedding resolves complex single-cell trajectories. Nat. Methods 14, 979-982 (2017).

88. Zhang, H. M. et al. AnimalTFDB 2.0: a resource for expression, prediction and functional study of animal transcription factors. Nucleic Acids Res. 43, D76-D81 (2015).

\section{ACKNOWLEDGEMENTS}

We thank Lucile Miquerol for providing the Gja5 $5^{E G F P /+}$ mice, Bo Zhou and Hui Cheng for providing the Kit// ${ }^{G F /+}$ mice, Yuqiang Ding for providing the Rosa ${ }^{\text {LoxP-Stop-LoxP-EYFP/+ }}$ mice, Aibin He for providing the Rosa ${ }^{\text {LoxP-Stop-LoxP-H2b-mCherry/+ }}$ mice. This work was supported by the National Key R\&D Program of China (2020YFA0112400, 2021YFA1100901，2016YFA0100601，2017YFA0103400, 2017YFA0102702), the National Natural Science Foundation of China (81890991, 31930054, 31625018, $31425012,31871173,81900115,82000111,91839301)$, the Program for Guangdong Introducing Innovative and Entrepreneurial Teams (2017ZT07S347), and the Key R\&D Program of Guangdong Province (2019B020234002).

\section{AUTHOR CONTRIBUTIONS}

Y. Lan, F.T., and B.L. conceived and designed the study; S.H., Z.C., and X.D. performed mouse VEC-related experiments including cell sorting, culture and immunostaining with help from S.L., Y. Li, B.W., Y.N. and X.N.; Y.G., Y. Hu, X.F., Y. Hou, X.L., and L.W. performed scRNA-seq; Z.L. and J.D. performed bioinformatics analysis; Y.Z. and Q.X. performed dissection and cell sorting of human embryos. B.Z. provided precious experimental materials and advices for the lineage tracing experiment. Y. Lan, F.T., and B.L. wrote the manuscript. All authors reviewed the manuscript.

\section{COMPETING INTERESTS}

The authors declare no competing interests.

\section{ADDITIONAL INFORMATION}

Supplementary information The online version contains supplementary material available at https://doi.org/10.1038/s41422-022-00615-z.

Correspondence and requests for materials should be addressed to Bing Liu, Fuchou Tang or Yu Lan.

Reprints and permission information is available at http://www.nature.com/ reprints

Open Access This article is licensed under a Creative Commons Attribution 4.0 International License, which permits use, sharing, adaptation, distribution and reproduction in any medium or format, as long as you give appropriate credit to the original author(s) and the source, provide a link to the Creative Commons license, and indicate if changes were made. The images or other third party material in this article are included in the article's Creative Commons license, unless indicated otherwise in a credit line to the material. If material is not included in the article's Creative Commons license and your intended use is not permitted by statutory regulation or exceeds the permitted use, you will need to obtain permission directly from the copyright holder. To view a copy of this license, visit http://creativecommons. org/licenses/by/4.0/.

(c) The Author(s) 2022 